\title{
Fe II emission lines in Be stars
}

\section{Empirical diagnostic of physical conditions in the circumstellar discs ${ }^{\star}$}

\author{
M. L. Arias ${ }^{1,3, \star \star}$, J. Zorec ${ }^{2}$, L. Cidale ${ }^{1,3}$, A. E. Ringuelet ${ }^{1}$, N. I. Morrell ${ }^{4}$, and D. Ballereau ${ }^{5}$ \\ 1 Facultad de Ciencias Astronómicas y Geofísicas, Universidad Nacional de La Plata, Paseo del Bosque s/n, (1900) La Plata, \\ Argentina \\ e-mail: mlaura@fcaglp.fcaglp.unlp.edu.ar \\ 2 Institut d'Astrophysique de Paris, UMR7095 CNRS, Université Pierre \& Marie Curie, 98bis Bd. Arago, 75014 Paris, France \\ 3 Instituto de Astrofísica de La Plata (CONICET-UNLP), Paseo del Bosque s/n, (1900) La Plata, Argentina \\ 4 Las Campanas Observatory, Carnegie Observatories, Casilla 601, La Serena, Chile \\ 5 GEPI, UMR 8111 du CNRS, Observatoire de Paris-Meudon, 92195 Meudon Cedex, France
}

Received 8 March 2006 / Accepted 22 June 2006

\section{ABSTRACT}

\begin{abstract}
Aims. The Fe II emission lines formed in the circumstellar envelopes (CE) of classical Be stars are studied in order to determine whether they are optically thin or optically thick. We also aim at deriving both average Fe II line excitation temperatures and the extent of their formation region in the CE.

Methods. We simultaneously observed several series of Fe II emission lines in the $\lambda \lambda 4230-7712 \AA$ wavelength interval and the first members of the hydrogen Balmer series of 18 southern classical Be stars. The optical depth regime that controls the formation of the observed Fe II lines and the physical parameters of their CE formation region were studied using the empirical self-aborption-curve (SAC) method.

Results. Our calculations give an average value of $\tau_{\mathrm{o}}=2.4 \pm 0.9$ for the optical depth of the studied Fe II lines, which implies that these lines are optically thick in the CE of Be stars. Qualitative indications that Fe II emission lines should be formed in circumstellar regions close to the central star are inferred from the correlations between Fe II emission line widths and $V$ sin $i$. The application of the SAC method to Fe II emission lines confirms this result, which gives $R_{\mathrm{e}}=2.0 R_{*} \pm 0.8$ for the extension of the line-forming region. The proximity of the line-forming region to the central star is also supported by the behavior of the source function of Fe II lines, which rapidly decreases with radii. This prevents the lines from being formed over extended regions and/or far from the star. Finally, the correlations of the central depression in the Balmer emission lines with $V \sin i$ are consistent with the flattened geometrical shapes of CEs.
\end{abstract}

Key words. stars: emission-line, Be - stars: circumstellar matter - line: profiles - line: formation

\section{Introduction}

The ionization potential of neutral $\mathrm{Fe}$ is $7.8 \mathrm{eV}$, while that of Fe II ions is $16.2 \mathrm{eV}$. This implies that due to the average excitation conditions that exist in the atmospheric and exophotospheric regions of a wide variety of stellar objects, iron is present mainly in the Fe II ionization state. In fact, Fe II lines are observed in the Sun, in Be stars and other objects with the $\mathrm{B}[\mathrm{e}]$ phenomenon, $\mathrm{Be} / \mathrm{X}$ stars, $\mathrm{LBV}$, cataclysmic variables, cool variables, novae, supernova remnants, HII regions, planetary nebulae, AGN, quasars, etc.

Although the Grotrian diagram of the Fe II ion is quite complex, its atomic levels can be classified into three categories: low even levels, metastable levels at roughly $3 \mathrm{eV}$ above the lower levels, and high odd levels at about $5 \mathrm{eV}$ from the lower or fundamental levels. Transitions among these levels then produce spectral lines that are seen in the UV, optical, and near IR spectral region. In each case, different regions of a given environment

^ Figures 7-17 and Tables 4-7 are only available in electronic form at http://www . aanda.org

$\star \star$ Visiting Astronomer of CASLEO, operated under agreement among CONICET and the National Universities of La Plata, San Juan and Córdoba, Argentina. can be responsible for the formation of the observed Fe II lines (Viotti 1976; Collin-Soufrin et al. 1979, 1980).

Emission in the first Balmer members can be seen in all sub-spectral types of Be stars, while emission in Fe II lines is mainly seen in subtypes earlier than B5 (Hubert-Delplace \& Hubert 1979). Qualitative descriptions of the occurrence of Fe II lines in Be stars were made by Wellman (1952), Viotti \& Koubský (1976), Geisel (1970), Allen \& Swings (1976), Viotti (1976), Slettebak (1982), and Polidan \& Peters (1976). Hanuschik $(1987,1988)$ and Ballereau et al. (1995) carried out somewhat more systematic studies, but they limited their discussion to the strongest Fe II emission lines in the visual. In these works the opacity regime that controls the formation of Fe II emission lines and the actual location and/or extent of their formation region have not been clearly established. All discussions by Hanuschik $(1987,1988)$ and Dachs et al. (1992) on the average kinematic properties of discs in Be stars and conclusions that Fe II lines are formed inside the $\mathrm{H} \alpha$ and $\mathrm{H} \beta$ emission lineformation zone are both entirely based on the assumption that Fe II lines are optically thin. In contrast, Ballereau et al. (1995) claim that Fe II lines in Be stars must be optically thick, which can lead to a somewhat different vision of the kinematics of $\mathrm{CE}$ and of the actual location of the formation region of these lines. 
In general, it is thought that this region is located in the $\mathrm{H} \mathrm{II} \rightarrow \mathrm{H} \mathrm{I}$ transition region (Netzer 1988) in a wide variety of astrophysical objects. Tarafdar \& Apparao (1994) argued that Fe II emission lines in Be stars cannot form in the H II region around the central object, because of its small extent.

It follows then that the location of the Fe II emission lineformation region in the $\mathrm{CE}$ of $\mathrm{Be}$ stars can be determined less ambiguously, if the optical depth regime of lines and the extent of their formation region are analyzed simultaneously. This is the precise aim of the present work. To this purpose, we used the self-absorption-curve (SAC) method developed by Friedjung \& Muratorio (1987), which enabled us to determine consistently the optical depth regime of the studied lines, their average excitation temperature, and the extent of their formation region. The method is based on the use of many Fe II-line multiplets, each with many emission lines.

\section{Stellar sample and observations}

We carried out observations of 18 southern Be stars at the Complejo Astronómico El Leoncito (CASLEO), San Juan, Argentina in March and September 1996, using the $2.15 \mathrm{~m}$ telescope, a REOSC échelle Cassegrain spectrograph with a $400 \mathrm{~mm}^{-1}$ grating in cross dispersion and a Tek $1024 \times$ 1024 CCD. The spectral range from $3900 \AA$ to $8000 \AA$, with mean resolution $R=11500$, was observed using two different tilts of the grating. Data reduction was made using the IRAF ${ }^{1}$ software package. Most of the program objects were observed in both above-mentioned epochs.

The studied objects are classical Be stars, i.e. non-supergiant B-type stars whose spectrum has, or had at some time, one or more Balmer lines in emission (Jaschek et al. 1981; Collins 1987). All selected Be stars show significant emission in the first terms of the hydrogen Balmer series and in the Fe II lines. As indicated in the introduction, most of these stars are then hotter than spectral type B5. Table 1 lists the program stars and their fundamental parameters and gives the log of observations. Julian days and detailed spectral ranges observed are given in the online Table 4 . When available, the MK spectral types and the $\left(\log T_{\text {eff }}, \log g\right)$ parameters are from the $\operatorname{BCD}\left(\lambda_{1}, D\right)$ system (Divan \& Zorec 1982; Frémat et al. 2005; Zorec et al. 2005). The $V \sin i$ are from Chauville et al. (2001). The inclination angle $i$ of each star is from Frémat et al. (2005), where this parameter was derived using models of stellar atmospheres that take into account the gravitational darkening effect induced by the geometrical distortion produced by the fast rotation of stars.

\section{Fe II emission line profiles}

We studied several Fe II line multiplets: 27, 28, 37, 38, 48, 49, 55,73 , and 74 . For rare cases, we could also measure some lines of multiplets 40,41 , and 43 . In the chosen multiplets the emission is the strongest, so much easier to identify and measure. The respective basic atomic data were taken from the National Institute of Standards and Technology (NIST) database (http://physics.nist.gov/cgi-bin/AtData/main_asd) and R. L. Kurucz (1995, private communication).

Figure 1 shows line emission profiles normalized to the continuum of a sample of Fe II line multiplets observed in some

1 IRAF is distributed by the National Optical Astronomy Observatories, which is operated by the Association of Universities for Research in Astronomy (AURA), Inc., under cooperative agreement with the National Science Foundation.
Table 1. Program stars and log of observations.

\begin{tabular}{rlrlccl}
\hline \hline Object & Sp.T & $\begin{array}{r}V \sin i \\
\mathrm{~km} \mathrm{~s}^{-1}\end{array}$ & $\log T_{\text {eff }}$ & $\log g$ & $\begin{array}{c}i \\
{[\mathrm{deg}]}\end{array}$ & Date \\
\hline HD 41335 & B1.5IIIne & 358 & 4.320 & 3.89 & 69 & 1,2 \\
HD 45725 & B2.5IV-Ve & 330 & 4.251 & 3.90 & 67 & 3 \\
HD 48917 & B2III-IVe & 205 & 4.308 & 3.40 & 45 & 3 \\
HD 50013 & B1.5IVne & 243 & 4.391 & 4.02 & 37 & 1,2 \\
HD 56139 & B2IVe & 84 & 4.291 & 3.62 & 17 & 3 \\
HD 58978 & B0Vpe & 375 & 4.388 & 4.15 & 55 & 3 \\
HD 63462 & B0Ve & 435 & 4.424 & 3.60 & 90 & 1,2 \\
HD 88661 & B2IVpne & 237 & 4.333 & 3.99 & 39 & 1,2 \\
HD 91465 & B3IIIne & 266 & 4.240 & 3.52 & 67 & 1,2 \\
HD 105435 & B2IVne & 258 & 4.349 & 3.92 & 42 & 1,2 \\
HD 110335 & B6IVe & $208^{a}$ & $4.120^{b}$ & $3.28^{b}$ & 63 & 3 \\
HD 112091 & B5Vne & 210 & 4.309 & 3.93 & 36 & 3 \\
HD 120991 & B2IIIe & 70 & 4.347 & 3.69 & 13 & 1,2 \\
HD 124367 & B4IVne & 295 & 4.243 & 3.76 & 63 & 1,2 \\
HD 148184 & B0.5Vpe & 144 & 4.459 & 3.91 & 20 & $1,2,4$ \\
HD 157042 & B2IVe & 340 & 4.338 & 4.06 & 53 & 4 \\
HD 158427 & B3Vne & 290 & 4.256 & 3.99 & 51 & 4 \\
HD 164284 & B2Ve & 262 & 4.426 & 3.95 & 53 & 4 \\
\hline
\end{tabular}

Dates: $1 \equiv 5$ Mar. 1996, $2 \equiv 6$ Mar. 1996, $3 \equiv 7$ Mar. 1996, $4 \equiv 21$ Sep. 1996. Julian days are given in the online Table 4. ${ }^{a}$ Yudin (2001); ${ }^{b}$ de Geus et al. (1989). The inclination angle $i$ was derived using stellar atmospheres calculated for rotationally deformed and gravitationally darkened stars (Frémat et al. 2005).

Be stars. The wavelength of the plotted Fe II lines is shown in the first box of the figure. Line $\lambda 5363$ corresponds to multiplet 48 , while the remaining ones are of multiplet 49 . Figure 1 is an excerpt of the spectroscopic data obtained. The profiles of most Fe II lines used to obtain the SAC curve of each object are available online (online Fig. 7 to Fig. 12). Although many lines were measured, not all of them are displayed in the atlas. Due to space limitations, we had to choose a layout presenting only the most outstanding among the observed transitions. All Fe II and hydrogen Balmer lines were observed simultaneously. For each star, the Balmer $\mathrm{H} \alpha, \mathrm{H} \beta$, and $\mathrm{H} \gamma$ lines are also shown in Fig. 1. The online atlas of line profiles also includes the first three lines of the hydrogen Balmer series (the online Figs. 13 to 15). The velocity scales used are heliocentric.

As seen in Fig. 1, all Fe II profiles are double peaked and have a central depression whose depth and shape is different from line to line. The Fe II lines are quite weak; in general they do not rise above some 0.2 in intensity over the continuum. Contrary to the hydrogen Balmer lines, they do not have extended wings. We can roughly distinguish two types of Fe II line emission profiles: a) fairly symmetrical (e.g. HD 45725 , HD 48917); b) asymmetrical, where one of the peaks is either more intense or wider (e.g. HD 50013). In a given object, most Fe II line emission profiles have a similar shape. There are, however, few objects where the central depression in the line profiles, or the relative intensity of peaks, change from one line to another, even if they have been observed simultaneously (HD 45725, HD 120991, HD 148184). This may suggest the presence of some inhomogeneity in the CE. Most objects show similar Fe II line profiles from one observing date to another. Fe II line emission profiles show similarities in their global shape i.e., central depression and relative intensity of the emission peaks to those of $\mathrm{H} \beta$ and $\mathrm{H} \gamma$ lines. Only in the extreme cases of pronounced asymmetries in Fe II lines do they also appear in $\mathrm{H} \alpha$. 


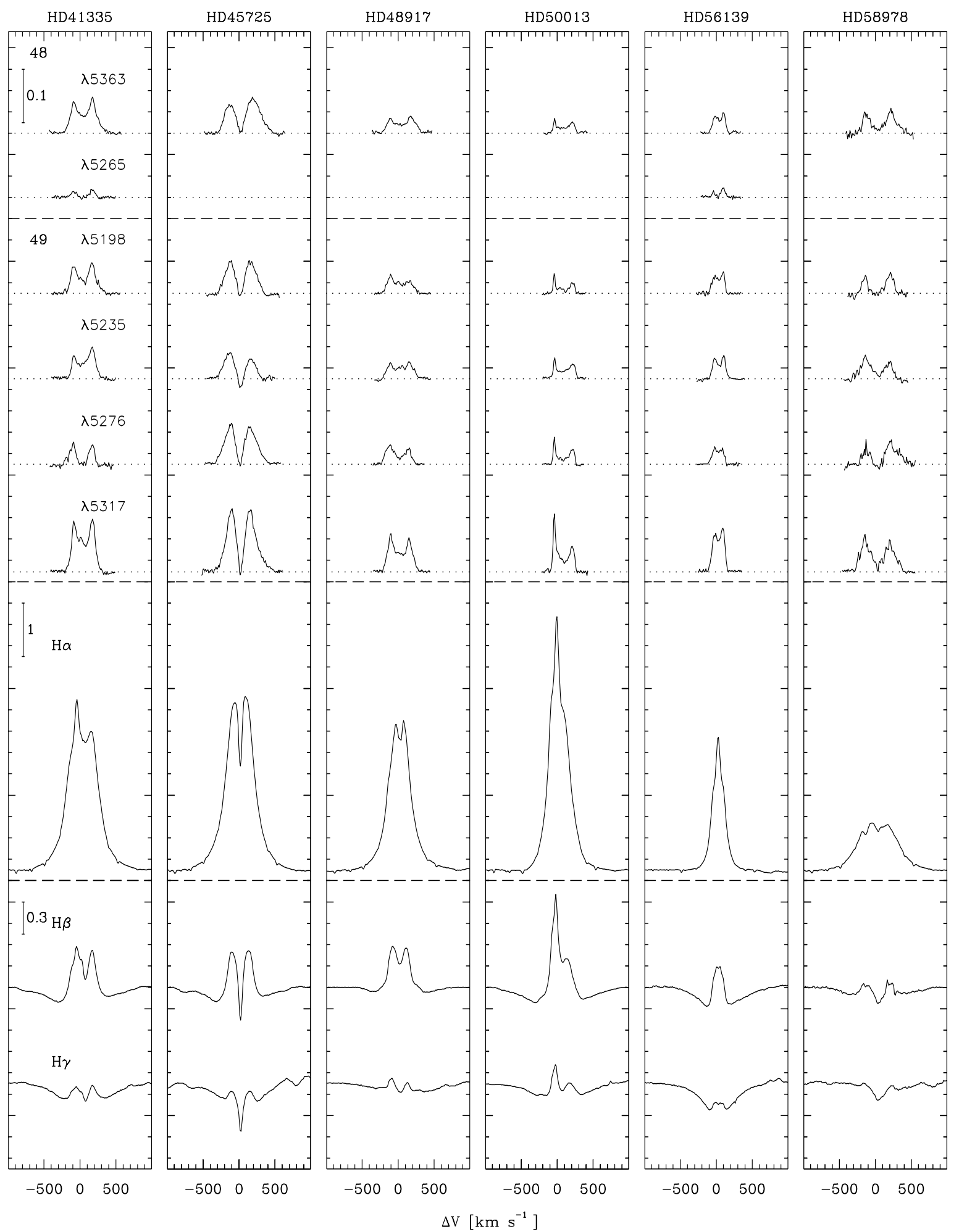

Fig. 1. Fe II and Balmer emission line profiles of some observed Be stars. The numbers in the upper corners of the left vertical panel of Fe II line profiles indicate the multiplet number to which the series of lines just below belong. The Fe II emission line profiles have all the same intensity scale, and $\mathrm{H} \beta$ and $\mathrm{H} \gamma$ lines have a common intensity scale, which is different for $\mathrm{H} \alpha$. Velocities in abscissas are heliocentric. See also the online catalogue of line profiles observed in other program stars.

\section{Measurements and correlations}

We performed the following measurements on each emission line profile: 1) central wavelength of lines, 2) intensity and velocity of the peaks and of the central depression, 3) line emission equivalent width $(W), 4)$ separation of emission peaks $\left(\Delta_{\mathrm{p}}\right)$,
4) widths at half intensity $\left(\Delta_{1 / 2}\right)$ and at intensity $I / I_{\mathrm{c}}=1.0\left(\Delta_{1}\right)$. As is known, some Fe II lines appear in the wings of stronger lines; for example, Fe II $\lambda 4351$ or Fe II of multiplet 42 are in the wings of $\mathrm{H} \gamma$ and $\mathrm{He}$ I lines, respectively. We measured those lines only if the underlying line wings were well-defined. All these measurements are available in the online Table 5. 
Figure 2 shows the equivalent widths $W$ of all Fe II lines as a function of the emission-peak separation $\Delta_{\mathrm{p}}$ in $\mathrm{km} \mathrm{s}^{-1}$, measured in four of the observed objects. The relation shown in Fig. 2 is representative of the behavior of $W$ against $\Delta_{\mathrm{p}}$ as seen in almost all studied objects; i.e. whatever the line strength of the Fe II line emissions in a given star, $\Delta_{\mathrm{p}}$, is the same within an average dispersion $\pm 50 \mathrm{~km} \mathrm{~s}^{-1}$. The same relation is also valid for line widths $\Delta_{1 / 2}$ and $\Delta_{1}$, although in the last case, points are somewhat more scattered due to the measurement difficulties/uncertainties related to $\Delta_{1}$. All $W$ vs. $\Delta_{\mathrm{p}}$ diagrams obtained are shown in the online Fig. 16.

If all Fe II lines were optically thin, this result would mean that there is a kinematically delimited formation region shared in the CE by all these lines. The average $\left\langle\Delta_{\mathrm{p}}\right\rangle$ value can then be used as the typical Fe II-line emission-peak separation, in particular, when this quantity needs to be studied as a function of another stellar property, like rotation. Figure 3 shows $\left\langle\Delta_{p}\right\rangle$ versus $V \sin i$ of program objects. In the same figure we also plot $\left\langle\Delta_{1 / 2}\right\rangle$ and $\left\langle\Delta_{1}\right\rangle$ as a function of $V \sin i$. The correlations obtained agree with results in previous works, although in most cases authors used a reduced number of individual Fe II lines from different multiplets (Hanuschik 1987, 1988; Ballereau et al. 1995; Slettebak et al. 1992). The dashed line in each box of Fig. 3 is the linear regression fit for which the slopes and correlation coefficients are:

$$
\left.\begin{array}{rl}
\overline{\Delta_{\mathrm{p}}} & =0.86 \times V \sin i+\quad 21 ; r=0.82 \\
\overline{\Delta_{1 / 2}} & =1.10 \times V \sin i+\quad 85 ; r=0.85 \\
\overline{\Delta_{1}} & =1.25 \times V \sin i+147 ; r=0.87
\end{array}\right\} .
$$

Due to uncertainties in determining the widths of individual lines, small differences may exist in the regression line coefficients obtained in previous works, because in the present estimation they correspond to the average widths obtained from many individual lines. In our case, the uncertainties of slopes are $\approx \pm 0.20$ on average. All these results can then be considered consistent. Since the slopes derived from average widths coincide with those of individual lines, it can be concluded that all Fe II lines share the same delimited formation region.

The dotted line added to correlations in Fig. 3 corresponds to $\Delta=2 V \sin i$. This line gives an upper limit to the expected rotational broadening of line profiles. Values $\left\langle\Delta_{1}\right\rangle \gtrsim 2 V \sin i$ would imply that line-broadening mechanisms other than rotation can be operating. A tight relation $\left\langle\Delta_{1}\right\rangle=2 V \sin i$ would mean coupling or co-rotation of the inner CE layers with the star. The corotation could be produced by magnetic fields whose presence might be suspected (Neiner et al. 2003; Neiner 2006).

All measurements carried out on Balmer emission lines were performed on profiles corrected for the underlying photospheric absorption component following the procedure applied by Chauville et al. (2001). These measurements are given in the online Table 6. As in previous works (cf. Andrillat \& Fehrenbach 1982; Dachs et al. 1986; Slettebak et al. 1992), the widths of Balmer lines correlate with $V \sin i$, except the width at intensity $I / I_{\mathrm{c}}=1.0$, which is not only more difficult to measure but can also be affected by several different broadening mechanisms, in particular, the electron scattering (Castor et al. 1970). We found, however, that, to our knowledge, it has not yet been shown so clearly in other previous attempts. We see that the equivalent width of the central depression, $W_{\mathrm{cd}}$, of $\mathrm{H} \beta, \mathrm{H} \gamma$, and $\mathrm{H} \delta$ emission lines show quite a well-defined trend defined with $V \sin i$ (average regression coefficients $r \simeq 80 \%$ ). This result is shown in Fig. 4. It appears then that the $\mathrm{CE}$ region producing the emission and the central top-absorption depression in the $\mathrm{H} \beta, \mathrm{H} \gamma$, and

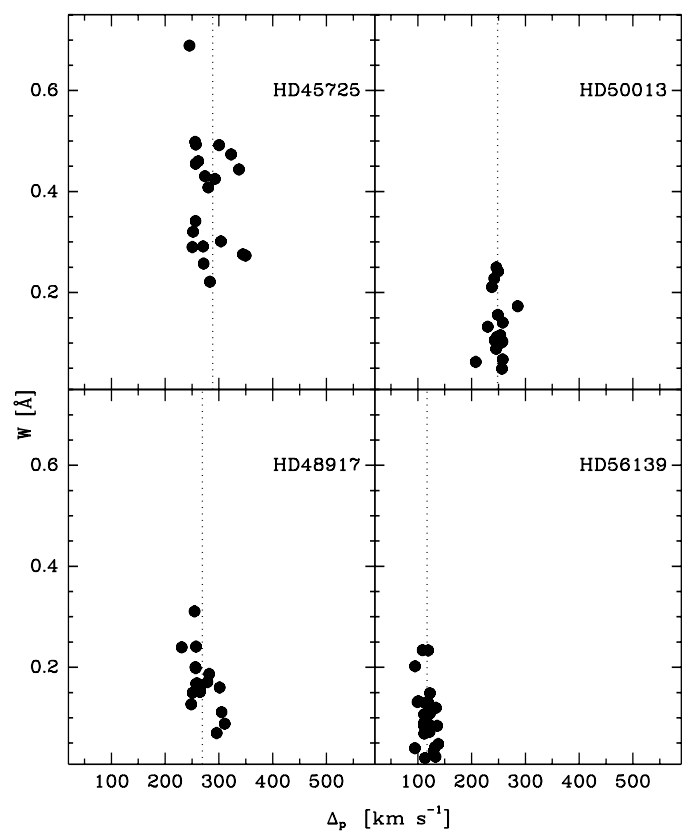

Fig. 2. Equivalent widths $W(\AA)$ of individual Fe II emission lines against their peak separation $\Delta_{\mathrm{p}}\left(\mathrm{km} \mathrm{s}^{-1}\right)$ in four observed Be stars. All $\Delta_{\mathrm{p}}$ are nearly the same for a given star, which suggests a common formation region. Vertical dotted lines indicate the average values of $\Delta_{\mathrm{p}}$. See also the online Fig. 16.

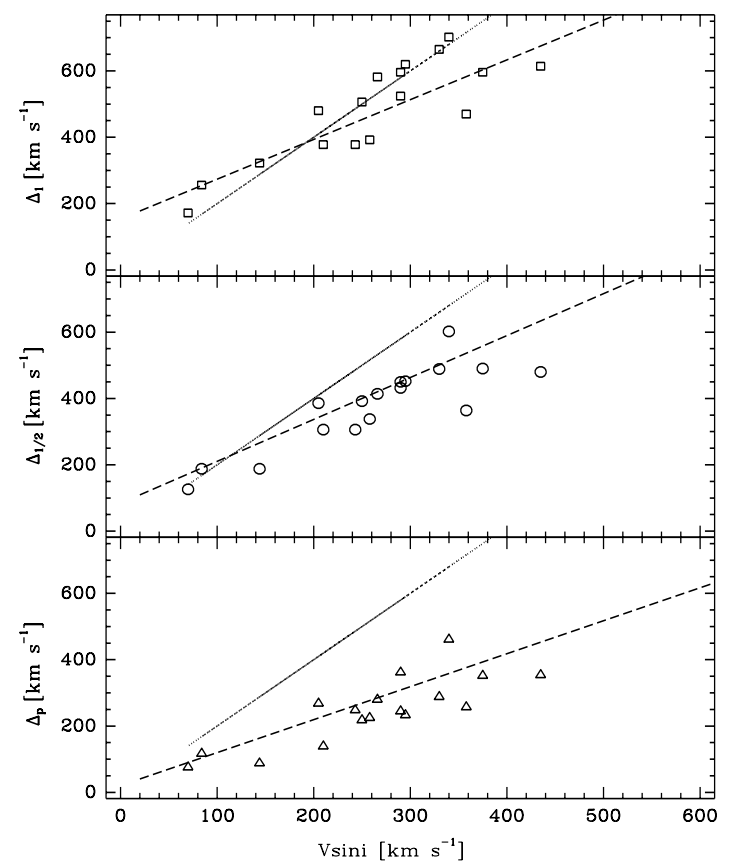

Fig. 3. Average widths of Fe II emission lines per star against the corresponding $V \sin i$. From top to bottom: $\left\langle\Delta_{1}\right\rangle=$ width at intensity $I / I_{\mathrm{c}}=1.0,\left\langle\Delta_{1 / 2}\right\rangle=$ width at half intensity, $\left\langle\Delta_{\mathrm{p}}\right\rangle=$ separation of peaks. The dotted line in each block represents $\Delta=2 V \sin i$. The dashed lines are the regressions given in (1).

$\mathrm{H} \delta$ lines is somewhat flattened. On the other hand, we note that the average inclination angle of the rotational axis of program Be stars is low, $\langle i\rangle=48^{\circ} \pm 21$ (see Table 1). Since the mentioned self absorptions in the Balmer line emission profiles do not have negligible equivalent widths, $W_{\text {cd }} \lessgtr 0.3 \AA$, discs must have non negligible optical depth in the perpendicular direction to the equator. 


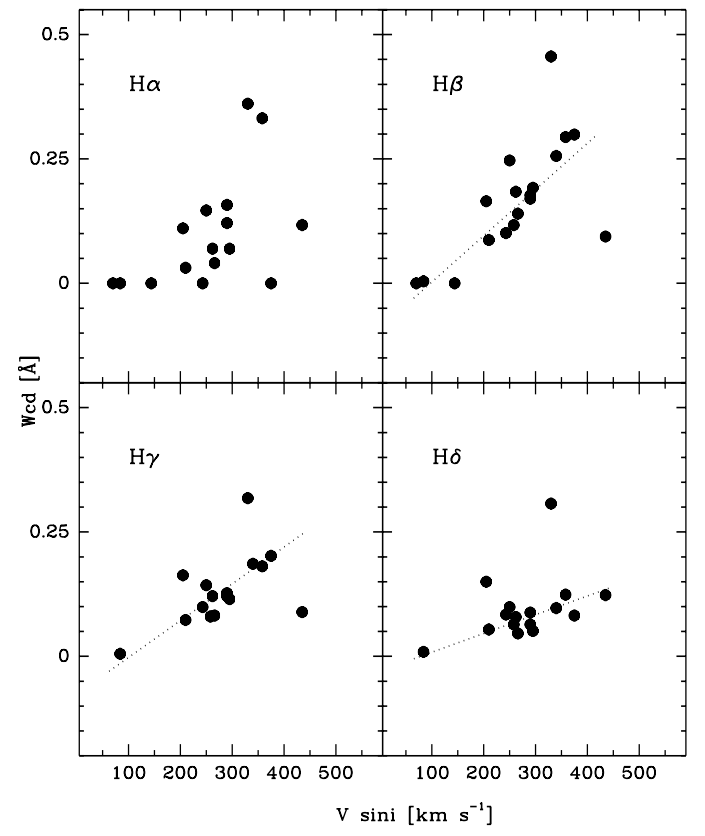

Fig. 4. Equivalent width of the central depression in Balmer emission lines $W_{\text {cd }}$ against the $V \sin i$. The dotted lines are only indicative of the observed trends. These trends suggest that the Balmer emission line formation region is somewhat flattened.

\section{The SAC method}

To analyze emission lines, Wellman (1952) and Viotti (1970) developed empirical methods similar to "curves-of-growth". Friedjung \& Malakpur (1971) proposed a different formalism that later became the self-absorption-curve method (SAC, Muratorio 1985; Friedjung \& Muratorio 1987). While the curveof-growth methods reveal effects related to the atomic level population in the emitting layers, the SAC method makes the opacity effect explicit on the emitted radiation intensity. It then carries information on the optical depth regime that controls the Fe II line emission formation in the CE of Be stars. The SAC method has been successfully applied to studying CE in a number of different types of objects: luminous blue stars (Muratorio \& Friedjung 1988, Muratorio et al. 1992), B[e] stars (Muratorio et al. 2002a), P Cygni (Muratorio et al. 2002b), novae (Selvelli \& Friedjung 2003), symbiotic stars (Kotnik-Karuza et al. 2002), the Be star component in Z CMa (van den Ancker 2004), etc. In this paper, we use the SAC method to determine the optical depth regime of Fe II emission lines observed in 17 Be classical stars and to estimate the extent of their formation region in the CE (HD 164284 has Fe II lines too small to be measured reliably).

The SAC method assumes that the emitting region is a flat disk with uniform density and temperature, which is characterized by the optical depth $\tau_{\mathrm{o}}$ in the center of a given spectral line. By comparing the empirical SAC with the theoretical one, we can derive the sought physical quantities. The theoretical SAC is defined by the relation $Y_{\lambda}=Y_{\lambda}\left(X_{\lambda}\right)$ where the respective variables $\left(X_{\lambda}, Y_{\lambda}\right)$ are defined as follows (Friedjung \& Muratorio 1987):

$$
\left.\begin{array}{rl}
X_{\lambda} & =\log \tau_{l}-\log \left(N_{l} / g_{l}\right)-C_{1} \\
Y_{\lambda} & =Q\left(\tau_{l}\right)+\log \left(N_{u} / g_{u}\right)+C_{2}
\end{array}\right\}
$$

where $\tau_{l}$ is the opacity in the central wavelength of the spectral line; $N_{u, l}$ and $g_{u, l}$ are the populations and statistical weights of the corresponding upper and lower atomic levels, respectively;

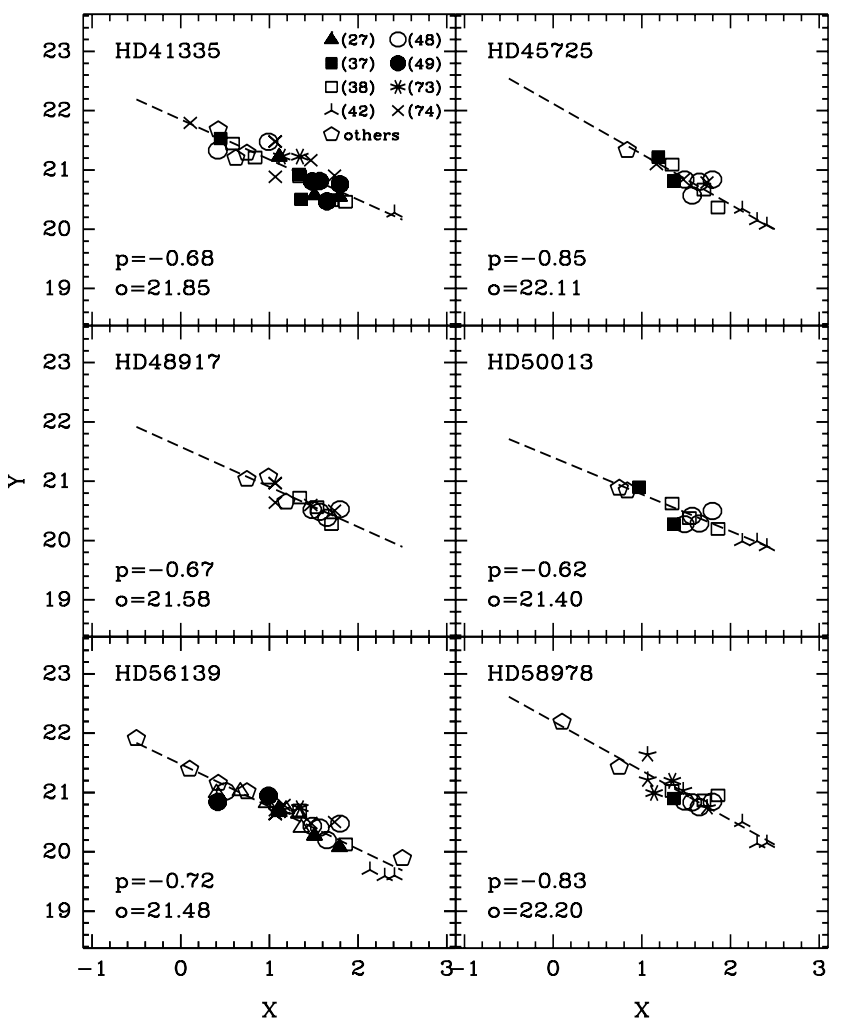

Fig. 5. Empirical SAC slopes for some stars whose lines are shown in Fig. 1. Each symbol corresponds to a given multiplet. The correspondence between symbols and multiplets is shown in the first left upper panel. In each panel are also given the slope $p=\partial Y / \partial X$ and $o=$ ordinate at $X=0$. The slopes indicate that Fe II lines are optically thick. Optically thin lines would produce slopes $p \approx 0$. See also the SAC of all program stars in the online Fig. 17.

$Q\left(\tau_{l}\right)$ is the theoretical SAC curve that measures the emitting efficiency of the studied environment and thus, it describes the self absorption in the spectral line; and $C_{1}$ contains atomic parameters, while $C_{2}$ depends on the area of the emitting region and on its characteristic velocity. The simplest form of the theoretical SAC function $Q\left(\tau_{l}\right)$ is:

$Q\left(\tau_{l}\right)=\log \left[\left(1-\mathrm{e}^{-\tau_{l}}\right) / \tau_{l}\right]$.

From (2) and (3) it follows that for optically thin lines $\left(\tau_{l} \ll 1\right)$ $Q\left(\tau_{l}\right) \simeq 0$, so that the corresponding SAC curves are horizontal lines $(\partial Y / \partial X=0)$. In contrast, optically thick lines $\left(\tau_{l} \ll 1\right)$ produce a nearly straight slope. In the limit $\tau_{l} \gg 1$ the slope of the SAC curve is $\partial Q / \partial \log \tau_{l} \rightarrow-1$. Since on average velocity rates in the CE are larger than the thermal velocity, to derive (3) it was assumed that the intrinsic line profile is rectangular. The use of more realistic atomic line profiles complicates considerably the aspect of $Q\left(\tau_{l}\right)$. However, it does not change its formal dependence on the opacity significantly (Friedjung \& Muratorio 1987). In this work we use relation (3). Some uncertainties related to this approximation are discussed in Sect. 7.

The empirical counterpart of (2) is given by:

$$
\left.\begin{array}{l}
X_{\lambda}=\log \left(g_{l} f_{u l} \lambda_{\mathrm{o}}\right) \\
Y_{\lambda}=\log \left(F_{u l} \lambda_{\mathrm{o}}^{3} / g_{l} f_{u l}\right)
\end{array}\right\},
$$

where $F_{u l}$ is the integrated flux in a given line, $f_{l u}$ is the oscillator strength of the transition, and $\lambda_{\mathrm{o}}$ the central wavelength of the line. The empirical SAC is constructed with individual $\left(X_{\lambda}, Y_{\lambda}\right)$ multiplet segments, assuming that all multiplets have the same 
function $Q\left(\tau_{l}\right)$, i.e. the same formation region. Horizontal displacements of SAC segments of those multiplets with a common lower level with respect to a chosen reference multiplet, as well as vertical displacements of multiplet segments with common upper levels, lead to an estimate of relative level populations and of the average line excitation temperature. Comparison of the recomposed empirical SAC with the theoretical curve $Q\left(\tau_{l}\right)$ gives the optical depth of the reference-line multiplet and the radius of the corresponding emitting region.

\section{Results}

The equivalent widths of the Fe II lines studied in this work are given in the online Table 5. The corresponding atomic data are in the online Table 7 . The continuum fluxes at the respective line frequencies as a function of the stellar fundamental parameters given in Table 1 are from the Kurucz (1992) LTE models atmospheres. As an example of SAC curves obtained, Fig. 5 shows those of objects referred to in Fig. 1. The whole set of SAC curves obtained in this work are shown in the online Fig. 17. The $\left(X_{\lambda}, Y_{\lambda}\right)$ slopes of all program stars were fitted with straight lines. Figure 5 also gives the respective values of slopes $(p)$ and $y$-intercept ordinates (o). The slopes obtained for all program stars range as:

$-0.5 \pm 0.1 \lesssim \partial Y_{\lambda} / \partial X_{\lambda} \lesssim-1.0 \pm 0.1$.

According to the discussion in Sect. 5, (5) clearly shows that the studied Fe II emission lines are optically thick. The individual values of $\partial Y / \partial X$ are listed in Table 2 . As no studied line is optically thin, the horizontal part of the SAC curves in Figs. 5 and 17 is missing.

Since we cannot determine the location of the flat part of the SAC, the matching of the empirical SAC with $Q\left(\tau_{l}\right)$ is not obvious. To evaluate the optical depth $\tau_{\mathrm{o}}$ of the reference Fe II line multiplet, we thus preferred to equate the empirical slopes obtained using (4) with the theoretical slope derived from (3):

$\left(\partial Y_{\lambda} / \partial X_{\lambda}\right)_{\text {empirical }}=\partial Q(\tau) / \partial \log \tau$.

Assuming that the atomic level populations do not depart from Boltzmann statistics, although the population can deviate from LTE conditions, the relative displacements of the SAC segments of different multiplets with a common upper or lower level can be written as: $|\Delta(X, Y)|=\left(\chi_{1}-\chi_{2}\right) 5040 / T_{\text {ex }}$, where $\chi_{1}$ and $\chi_{2}$ are the excitation potentials of two given levels and $T_{\mathrm{ex}}$ is the corresponding excitation temperature. However, when the excitation temperature is high, as in program stars, these displacements are small and the determination of $T_{\mathrm{ex}}$ is difficult. We then preferred to determine $T_{\mathrm{ex}}$ by using an alternative iteration process. To this purpose, we defined the following variables $U$ and $V$ :

$$
\left.\begin{array}{l}
U=X_{\lambda}+Y_{\lambda}-\log \left(1-\mathrm{e}^{-\tau}\right) \\
V=\chi-\chi_{\mathrm{o}} \\
\tau=\tau_{\mathrm{o}} 10^{\frac{5040}{T_{\mathrm{ex}}}\left(\chi-\chi_{\mathrm{o}}\right)} 10^{\left(X_{\lambda}-X_{\mathrm{o}}\right)}
\end{array}\right\},
$$

which determine a straight line $U=U(V)$ whose slope, controlled with the $\chi^{2}$ test, is $5040 / T_{\mathrm{ex}}$. According to the star, we used as reference multiplet the one with the average wavelength lieing roughly in the middle of the re-composed SAC (frequently multiplet 38). Finally, to estimate the emitting region extent, we used the explicit form of the SAC obtained from relations (2) and (4) (Friedjung \& Muratorio (1987):

$\mathcal{S}=\frac{1}{2 \pi h c}\left[\frac{10^{-Q\left(\tau_{\mathrm{o}}\right)}}{\tau_{\mathrm{o}}}\right] \frac{F_{\mathrm{c}} W_{\lambda} \lambda^{4}}{V_{\mathrm{o}}} \mathrm{e}^{1.44 / \lambda T_{\mathrm{ex}}}$
Table 2. Parameters of the Fe II line emission formation derived from the SAC curves.

\begin{tabular}{rrrrrcrc}
\hline \hline Object & $\partial Y / \partial X$ & $\tau_{\mathrm{o}}$ & $T_{\mathrm{ex}}$ & $V_{\mathrm{o}}$ & $R_{\mathrm{e}} / R_{*}$ & \multicolumn{2}{c}{$R_{\mathrm{H}} / R_{*}$} \\
\cline { 7 - 8 } & $\mathrm{SAC}$ & & $\mathrm{K}$ & $\mathrm{km} \mathrm{s}^{-1}$ & $\mathrm{SAC}$ & $j=1$ & $j=0.5$ \\
\hline HD 41335 & -0.68 & 2.0 & 7600 & 182 & 2.0 & 2.8 & 7.8 \\
HD 45725 & -0.85 & 3.1 & 4500 & 245 & 4.2 & 2.3 & 5.3 \\
HD 48917 & -0.67 & 2.0 & 5000 & 193 & 2.3 & 1.5 & 2.3 \\
HD 50013 & -0.62 & 1.7 & 8800 & 153 & 1.1 & 2.0 & 3.8 \\
HD 56139 & -0.72 & 2.2 & 7000 & 94 & 1.7 & 1.4 & 2.1 \\
HD 58978 & -0.83 & 2.9 & 11600 & 245 & 1.3 & 2.1 & 4.5 \\
HD 63462 & -0.94 & 4.3 & 13300 & 247 & $*$ & 2.5 & 6.0 \\
HD 88661 & -0.95 & 4.5 & 6000 & 216 & 1.5 & 1.6 & 2.6 \\
HD 91465 & -0.73 & 2.2 & 5500 & 207 & 2.8 & 2.0 & 3.8 \\
HD 105435 & -0.49 & 1.2 & 5900 & 169 & 2.2 & 2.3 & 5.3 \\
HD 110335 & -0.65 & 1.8 & 5400 & 196 & 1.9 & 2.3 & 5.3 \\
HD 112091 & -0.72 & 2.2 & 7100 & 153 & 1.1 & 3.0 & 9.0 \\
HD 120991 & -0.65 & 1.8 & 6500 & 63 & 1.8 & 1.8 & 3.4 \\
HD 124367 & -0.83 & 2.9 & 5300 & 226 & 3.0 & 2.5 & 6.4 \\
HD 148184 & -0.69 & 2.0 & 12000 & 94 & 1.1 & 3.3 & 10.7 \\
HD 157042 & -0.65 & 1.8 & 6900 & 301 & 1.7 & 1.5 & 2.2 \\
HD 158427 & -0.61 & 1.7 & 6000 & 225 & 1.5 & 2.4 & 5.6 \\
\hline
\end{tabular}

$\tau_{\mathrm{o}}=$ average optical depth of Fe II lines in multiplet 38; $T_{\mathrm{ex}}=$ average excitation temperature of Fe II lines; $V_{\mathrm{o}}=$ characteristic emission line width in velocity units; $R_{\mathrm{e}} / R_{*}=$ radius of the Fe II line formation region derived with the SAC; $R_{\mathrm{H}} / R_{*}=$ radius derived with Huang's (1972) relation for optically thin lines.

$*: R_{\mathrm{e}} / R_{*} \approx\left(R / R_{*}\right) / \sqrt{\cos i}\left(R / R_{*} \approx 1, i \sim 90^{\circ}\right)$.

Note: HD 164284 has too tiny Fe II emission lines to perform any reliable measurement.

where $S$ is the surface of the emitting region, $V_{\mathrm{o}}$ is a characteristic emission line width in velocity units, $F_{\mathrm{c}}$ the flux of the continuum spectrum at the given line wavelength, and $W_{\lambda}$ the line equivalent width. Using $\tau_{\mathrm{o}}$ issued from (6) and $T_{\mathrm{ex}}$ iterated in (7), from relation (8), we obtain $\mathcal{S}=\pi\left(R_{\mathrm{e}}^{2}-R_{*}^{2}\right)$. However, as the "observed" emitting surface is aspect-angle-dependent, to calculate the radius $R_{\mathrm{e}} / R_{*}$ of the line emission formation region, we have corrected the area derived from (8) using $\mathcal{S} \rightarrow \mathcal{S} \cos i$. The inclination angle $i$ used in this correction is given in Table 1 . We note that since the effect of $i$ on the estimate of $R_{\mathrm{e}} / R_{*}$ is proportional to $1 / \sqrt{\cos i}$, the uncertainties on $i$ do not introduce sensitive changes in $R_{\mathrm{e}} / R_{*}$. In (8) it is implicitly assumed that $\mathrm{e}^{1.44 / \lambda T_{\mathrm{ex}}} \gg 1$. However, we do not obtain qualitatively different results for $\tau_{\mathrm{o}}$ and $R_{\mathrm{e}} / R_{*}$ when dropping this approximation. All quantities describing the Fe II emission region obtained in the present section are given in Table 2. For comparison, in this table the radii of the Fe II region calculated from Huang's (1972) expression are also given: $R_{\mathrm{H}} / R_{*}=\left(2 V \sin i / \Delta_{\mathrm{p}}\right)^{1 / j}(j=1 / 2$ for Keplerian rotation; $j=1$ for rotation with conservation of angular momentum), which actually should be used only for optically thin lines.

\section{Discussion}

In this section we briefly discuss three different, but related issues: the incidence of the line opacity regime on the estimate of the extent of the line-formation zone, formulation of the SAC by taking into account the optical depth in the line source function, and the interpretation of the line excitation temperature, which in the SAC method does not straightforwardly relate to the physical properties of the line-formation region. 


\subsection{Opacity of the Fe II line emission formation region}

One of the main results in this work is that the Fe II emission lines in the $\mathrm{CE}$ of the studied classical Be stars are optically thick. This means that models of Fe II line emission formation in Be stars, which can help to diagnose the physical properties of CE more precisely, must take the optical depth effects in these lines into account. The values of optical depths obtained are on average $\left\langle\tau_{\mathrm{o}}\right\rangle=2.4 \pm 0.9$. Even though uncertainties may be affecting the estimate of individual $\tau_{\mathrm{o}}$ values, the empirical SAC curves of Fig. 5 and those given in the online Fig. 17, show that slopes are far from being horizontal lines, which would be the case if $\mathrm{Fe}$ II lines were optically thin. The temperature structure of the CE in $\gamma$ Cas and 1 Del between 1 and 2 stellar radii derived by Jones et al. (2004) is also consistent with optically thick Fe II lines, which could otherwise act as an efficient cooling agent.

The extent of the Fe II emission-line formation region we obtained is on average $\left\langle R_{\mathrm{e}} / R_{*}\right\rangle=2.0 \pm 0.8$. It is then systematically smaller than the one obtained from Huang's (1972) relation with $j=0.5:\left\langle R_{\mathrm{H}} / R_{*}\right\rangle_{j=0.5}=5.1 \pm 2.4$, valid only for optically thin lines. From (8), where $R_{\mathrm{e}} / R_{*} \sim\left(1-\mathrm{e}^{-\tau_{\mathrm{o}}}\right)^{-1}$, we can see that the smaller is $\tau_{\mathrm{o}}$ the larger $R_{\mathrm{e}} / R_{*}$ becomes, in accordance with Huang's estimates that are valid for $\tau_{\mathrm{o}} \rightarrow 0$. Relation (8) can be reformulated for optically thin lines by considering that in this case $W_{\lambda} \sim \sqrt{\pi} \tau_{\mathrm{o}} \Delta \lambda_{\mathrm{D}}$, where $\Delta \lambda_{\mathrm{D}}=$ thermal Doppler line width. For some stars the SAC radii given in Table 2 are of the same order of magnitude as those obtained form Huang's (1972) formula. This is probably due to the uncertainties related to the $T_{\mathrm{ex}}$ determination.

In the present discussion we compare radii issued from two different formulations. On the one hand, there is relation (8) that ignores details on the kinematical properties of regions where the lines are formed. On the other, there is Huang's (1972) relation, which is based only on the kinematical aspects of the CE. However, several contributions have shown that the separation of emission peaks is a function of the velocity fields and the CE optical depth. Cidale \& Ringuelet (1989) found that in static CE the separation of the emission peaks is wider when the value of the optical depth $\tau_{\mathrm{o}}$ is higher. On the contrary, in moving, optically thick CE the interplay of opacity and velocity fields can lead to a reduction of the emission peak separation as $\tau_{\mathrm{o}}$ increases (Hummel 1994; Arias 2004; Arias et al. 2004, 2006).

\subsection{The line source function and the SAC}

In circumstellar layers where the Fe II line emissions are formed, $R \lesssim 2 R_{*}$, the temperature can be estimated assuming that the only energy input is from the geometrically diluted stellar radiation filed. Moujtahid et al. (2000) show that this approximation is valid for a $\mathrm{CE}$ close to the star:

$T_{\mathrm{CE}} \approx W(R)^{1 / 4} T_{\mathrm{eff}}$

where $W(R)$ is the geometrical dilution factor. In radii $R \lesssim 2 R_{*}$, $T_{\mathrm{CE}}$ is nearly the same as the thin disc temperature $T_{\mathrm{D}}$ derived using more detailed models (Carciofi \& Bjorkman 2006). Although the distances of the Fe II emission line regions derived in the present work are short, the excitation temperatures $T_{\text {ex }}$ are on average $30 \%$ smaller than $T_{\mathrm{CE}}$ given by (9). This reveals that non-LTE effects control the population of atomic levels in the line transitions. The level-populations are currently written as $N_{u, l}=b_{u, l} N_{u, l}^{*}$ (sub-indices " $u, l$ ' stand for "upper" and "lower" level), $b_{u, l}$ are the respective non-LTE deviation coefficients and $N_{u, l}^{*}$ are the level populations in LTE. By definition it

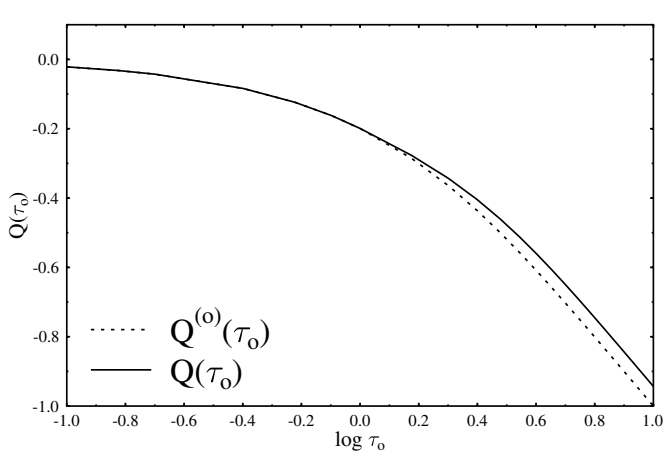

Fig. 6. Comparison of the SAC curve $Q\left(\tau_{\mathrm{o}}\right)$ given by (12) with $Q^{(\mathrm{o})}\left(\tau_{\mathrm{o}}\right)$ calculated in (3).

is $\Delta \chi / T_{\mathrm{ex}}=\Delta \chi / T_{\mathrm{CE}}-\ln \left(b_{u} / b_{l}\right)$, where $\Delta \chi=\chi_{u}-\chi_{l}$ is the difference of excitation potentials. We would then expect roughly that $b_{u} / b_{l} \sim \mathrm{e}^{-0.6 \Delta \chi / T_{\mathrm{CE}}}$, which implies that $b_{u} \neq b_{l}$. This also means that the source function $S_{\lambda}$ of each line is decoupled from the local Planck function $B_{\lambda}\left(T_{\mathrm{CE}}\right)$. As in this case there must be non-local feeding of energy, the source function becomes dependent on the line optical depth (Mihalas 1978, Chap. 11). The line source function $S_{\lambda}\left(\tau_{\mathrm{o}}\right)$ can then be approximately written as (Cidale \& Ringuelet 1989):

$S_{\lambda}\left(t_{\mathrm{o}}\right)=\left\{\begin{array}{r}S_{\mathrm{o}} \text { for } t_{\mathrm{o}} \lesssim 1 \\ S_{\mathrm{o}} \times t_{\mathrm{o}}^{q} \text { for } t_{\mathrm{o}}>1,\end{array}\right.$

where the factor $S_{\mathrm{o}}$ depends on the nature of the source function (see next section) and $q$ depends on whether the intrinsic lineabsorption profile is of Lorentz type, or Gaussian: $0.28 \lesssim q \lesssim$ 0.6 . To derive the SAC function $Q\left(\tau_{\mathrm{o}}\right)$ that reflects the opacity dependence of the source function given in (10), we write the flux per unit wavelength produced by a CE of total optical depth $\tau_{\mathrm{o}}$ as:

$F_{\mathrm{o}}=\mathcal{S} \int_{0}^{\tau_{\mathrm{o}}} S_{\mathrm{o}}\left(t_{\mathrm{o}}\right) \mathrm{e}^{-t_{\mathrm{o}}} \mathrm{d} t_{\mathrm{o}}$

where $\mathcal{S}$ is the area of the emitting surface projected in the observer's direction. Considering $S_{\mathrm{o}}=B_{\mathrm{o}}\left(T_{\mathrm{ex}}\right)=$ constant, the integration of (11) leads to the function $Q\left(\tau_{\mathrm{o}}\right)$ given by (3) (Friedjung \& Muratorio 1987) and used in the present work. $T_{\text {ex }}$ is the excitation temperature of the Fe II lines, which is assumed to be the same for all studied lines. However, adopting $S_{\mathrm{o}}=B_{\lambda}\left(T_{\mathrm{ex}}\right)$ as before, but considering the opacity-dependence of the source function indicated in (10), from (11) we obtain:

$10^{Q\left(\tau_{\mathrm{o}}\right)}= \begin{cases}\left(1-\mathrm{e}^{-\tau_{\mathrm{o}}}\right) / \tau_{\mathrm{o}} & \text { for } \tau_{\mathrm{o}} \lesssim 1 \\ {\left[\left(1-\mathrm{e}^{-\tau_{\mathrm{o}}}\right)+\int_{1}^{\tau_{\mathrm{o}}}\left(t^{q}-1\right) \mathrm{e}^{-t} \mathrm{~d} t\right] / \tau_{\mathrm{o}}} & \text { for } \tau_{\mathrm{o}}>1 .\end{cases}$

In Fig. 6 the SAC function $Q\left(\tau_{\mathrm{o}}\right)$ defined in (12) is compared with $Q^{(\mathrm{o})}\left(\tau_{\mathrm{o}}\right)$ given by $(3) . Q\left(\tau_{\mathrm{o}}\right)$ has similar properties as $Q^{(\mathrm{o})}\left(\tau_{\mathrm{o}}\right)$, i.e. $Q\left(\tau_{\mathrm{o}} \ll 1\right) \rightarrow 0$ and $\partial Q / \partial \ln \tau \rightarrow-1$ for $\tau_{\mathrm{o}} \gg 1$. However, for $\tau_{\mathrm{o}} \gtrsim 1$ a given slope $\partial Q\left(\tau_{\mathrm{o}}^{\text {new }}\right) / \partial \log \tau=$ $\partial Q^{(\mathrm{o})}\left(\tau_{\mathrm{o}}^{\text {old }}\right) / \partial \log \tau$ implies $\tau_{\mathrm{o}}^{\text {new }}>\tau_{\mathrm{o}}^{\text {old }}$, as is shown in the following table, where the slopes due to "old" opacities are reinterpreted with the "new" SAC curve:

\begin{tabular}{lllllllllrl}
\hline \hline & \multicolumn{10}{c}{$\tau_{\mathrm{o}}$} \\
\hline "old" & 1.0 & 2.0 & 3.0 & 4.0 & 5.0 & 6.0 & 7.0 & 8.0 & 9.0 & 10.0 \\
"new" & 1.3 & 2.6 & 3.8 & 4.9 & 6.0 & 7.0 & 8.1 & 9.1 & 10.2 & 11.0 \\
\hline
\end{tabular}




\subsection{The CE line-emitting power}

In the present formulation of the SAC, all information on the nature of the Fe II-line source function and on its relation to the physical properties of the line formation region is hidden in the $T_{\text {ex }}$ parameter. To inquire in what way the SAC method can be improved to draw some information from $T_{\mathrm{ex}}$, let us write the line source function $S_{\lambda}$ in the two level-atom approximation (Thomas 1965, 1983; Mihalas 1978):

$S_{\lambda}=\left[\int_{0}^{\infty} \phi_{\lambda} J_{\lambda} \mathrm{d} \lambda+\epsilon B_{\lambda}\left(T_{\mathrm{CE}}\right)+\eta B_{\lambda}\left(T_{r}\right)\right] /[1+\epsilon+\eta]$

where $\phi_{\lambda}$ is the intrinsic line absorption profile; $J_{\lambda}$ is the angleaveraged intensity of the radiation field; $\epsilon$ depends on the upper level collision-depopulating rates; $\eta$ represents the depopulation rate of the upper level by photoionization processes; $B_{\lambda}\left(T_{\mathrm{CE}}\right)$ is the Planck function for the local electronic temperature $T_{\mathrm{e}}=T_{\mathrm{CE}} ; T_{r}$ is a "radiation temperature", whose value is set by the photoinization and recombination rates. The source function can be collision-dominated if $\epsilon>\eta$ and $\epsilon B\left(T_{\mathrm{CE}}\right)>\eta B\left(T_{r}\right)$, radiation-dominated if $\epsilon<\eta$ and $\epsilon B\left(T_{\mathrm{CE}}\right)<\eta B\left(T_{r}\right)$, and mixeddominated in other compararative combinations of these terms. According to the source function nature, the $S_{\mathrm{o}}$ factor in (10) becomes:

$S_{\mathrm{o}} \approx \begin{cases}\epsilon^{1 / 2} B_{\lambda}\left(T_{\mathrm{CE}}\right) & \text { for collision-dominated } \\ \eta^{1 / 2} B_{\lambda}\left(T_{r}\right) & \text { for radiation-dominated }\end{cases}$

Thus, depending on the spectral line, local electron temperature, electron density, and the radiation field, $T_{\mathrm{ex}}$ reflects either the local electron temperature (collision-dominated), the stellar radiation field (radiation-dominated), or some combination of both (mixed-domination) that would be important to specify in further attempts of studying Fe II lines. By considering only the leading factors of the intervening radiative and collisional rates in $\epsilon$, $\eta$, and $B\left(T_{r}\right)$, we can determine the nature of the source function and its incidence on the values of $T_{\mathrm{ex}}$. This can also help to appreciate the relation between the value of $T_{\mathrm{ex}}$ and the radius derived of the Fe II line-formation region. Let us then write

$$
\begin{aligned}
& \epsilon \simeq C_{u l} / A_{u l} \\
& \left.\begin{array}{rl}
\epsilon & \simeq C_{u l} / A_{u l} \\
\eta & \simeq R_{u k} / A_{u l} \\
B\left(T_{r}\right) & \simeq \frac{2 h v_{u l}^{3}}{c^{2}}\left[\mathrm{e}^{h v_{u l} / k T_{\mathrm{e}}} \frac{R_{u k}}{R_{l k}} \frac{R_{k l}}{R_{k u}}-1\right]^{-1}
\end{array}\right\},
\end{aligned}
$$

where $C_{u l}$ are the collisional rates, $R_{n k}$ and $R_{k n}$ are respectively the radiative ionization and recombination rates to the $n$ atomic level, and $A_{u l}$ is the spontaneous emission rate. To calculate $R_{n k}$, we approximate the photoionization radiation field with $J_{v}=W(R) B_{v}\left(T_{\text {eff }}\right)$ (Vinicius et al. 2005). Table 6 gives the average source function parameters normalized to the local continuum flux $F_{\mathrm{c}}$. They were calculated using lines of multiplets 27, 38, 49, and the following parameters for typical Be stars (spectral type B1-B2): $T_{\text {eff }}=25000 \mathrm{~K}$, electron density $N_{\mathrm{e}}=3 \times 10^{12} \mathrm{~cm}^{-3}$, electron temperature $T_{\text {CE }}$ derived with (9).

Values in Table 6 show that the source function varies from genuine radiation-dominated at $R / R_{*}=1.5$ to mixed-dominated in $R / R_{*}=5.0\left[\eta \sim \epsilon\right.$ and $\left.\eta^{1 / 2} B\left(T_{r}\right) / F_{\mathrm{c}} \sim \epsilon^{1 / 2} B\left(T_{\mathrm{CE}}\right) / F_{\mathrm{c}}\right]$. The lower block of Table 7.3 shows the dependence of $T_{\mathrm{ex}}$ on distance $R$, which implies that $T_{\text {ex }}<T_{\mathrm{CE}} \ll T_{\text {eff. }}$. Thus, whenever $T_{\text {ex }}$ is low compared to $T_{\text {eff }}$, as it is for values in Table 5, it does not mean that the formation region of Fe II emission lines lies far from the central star. Since the source function is radiationdominated in regions of its maximum emission effectiveness, the effect of the electron temperature on the production of Fe II lines is marginal, as it acts through negligible collisional terms.
Table 3. Fe II line source function parameters.

\begin{tabular}{ccccc}
\hline \hline$R / R_{*}$ & $\epsilon$ & $\epsilon / \eta$ & $\epsilon^{1 / 2} B\left(T_{\mathrm{CE}}\right) / F_{\mathrm{c}}$ & $\eta^{1 / 2} B\left(T_{r}\right) / F_{\mathrm{c}}$ \\
\hline 1.5 & $0.03 \pm 0.01$ & $0.07 \pm 0.03$ & $0.06 \pm 0.01$ & $0.26 \pm 0.17$ \\
3.0 & $0.05 \pm 0.02$ & $0.35 \pm 0.09$ & $0.02 \pm 0.01$ & $0.06 \pm 0.02$ \\
5.0 & $0.05 \pm 0.02$ & $0.81 \pm 0.20$ & $0.01 \pm 0.00$ & $0.01 \pm 0.00$ \\
\hline$R / R_{*}$ & & $T_{\mathrm{CE}}(\mathrm{K})$ & $S_{\mathrm{o}} / F_{\mathrm{c}}$ & $T_{\mathrm{ex}}(\mathrm{K})$ \\
\hline 1.5 & & 14900 & $0.26 \pm 0.14$ & $12400_{-3400}^{+2600}$ \\
3.0 & & 9800 & $0.06 \pm 0.02$ & $8210_{-800}^{+650}$ \\
5.0 & & 7800 & $0.01 \pm 0.00$ & $5930_{-40}^{+10}$ \\
\hline
\end{tabular}

Finally, an explanation of the low values of radii $R / R_{*}$ derived with the SAC is given by the rapid decrease with distance of $S_{\mathrm{o}}=\eta^{1 / 2} B\left(T_{r}\right)$, as seen in Table 6 , which indicates that the Fe II emission-line formation zone in the CE cannot be very extended, or that it cannot be far from the central star. Short radii of the Fe II emission-line formation zone in Be stars $R_{\mathrm{e}} \sim 1.5 R_{*}$ and low line-excitation temperatures ranging from 4500 to $6000 \mathrm{~K}$ have also been recently found by Brusasco \& Cidale (2006, in preparation) using detailed non-LTE models.

\section{Conclusions}

We have performed an empirical analysis of the Fe II emission lines in Be stars to derive insights into the optical depth regime that characterizes these lines (optically thin or optically thick), as well as to obtain the average excitation temperature and the extent of their formation region in the CE.

We have presented observations of several series of Fe II line emission multiplets in the $\lambda \lambda 4230-7712 \AA$ wavelength interval and the first three members of the hydrogen Balmer series, which were observed simultaneously in 18 southern Be stars. Although Fe II lines in Be stars have already been studied by several authors, most of them considered only the strongest lines in different multiplets. On the contrary, the present analysis is based on the use of a large number of Fe II line multiplets. Observations were carried out for enough Be stars to render the obtained statistical insights reliable.

The correlations between the Fe II emission-line widths and $V \sin i$ suggest that the line formation region in the circumstellar disc cannot be situated far from the central star. On the other hand, we found a rather well-defined correlation between the central depression in the Balmer emission lines with the $V \sin i$, which indicates that CE have globally flattened geometrical structures.

In the present paper we analyze only the Fe II emission lines in detail. In contrast to previous works on the Fe II lines in Be stars, where it is systematically assumed that they are optically thin, we have made allowance for their possibly optically-thick character. The Fe II emission lines were thus studied using the self-absorption-curve (SAC) method. This analysis leads us to conclude that $\mathrm{Fe}$ II emission lines in Be stars are optically thick and that the optical depth in the line center is on average $\tau_{\mathrm{o}}=$ $2.4 \pm 0.9$. It has also been obtained that the line formation region lies on average near the central star, within $R / R_{*}=2.0 \pm 0.8$.

Considering the collision- and radiation-dependent terms of the line source function, we confirm that due to its rapid decrease with the radius, the Fe II line-emission formation region cannot be neither extended nor located far from the star.

Due to the non-LTE effects, the optical-depth dependence of the Fe II line source function should be taken into account explicitly in further improvements to the SAC method. In general, 
to gain precision in the derived physical parameters, the empirical methods for studying Fe II emission lines need to consider: 1) the opacity regime of lines; 2) their absorption line profile; 3 ) the nature of the source function regarding the processes determining the excitation of atomic levels, 4) the velocity field in the formation region. This is the aim pursued in a forthcoming paper, where new series of observations of Fe II lines will complete the study.

Acknowledgements. We would like to thank Dr. J. Chauville for his help in the reduction of some of the data. We warmly thank the comments and suggestions formulated by the referee.

\section{References}

Allen, D. A., \& Swings, J. P. 1976, A\&A, 47, 293

Arias, M. L. 2004, Ph.D. Thesis, University of La Plata, Argentina

Arias, M. L., Zorec, J., \& Ringuelet, A. 2004, BAAA, 47, 161

Arias, M. L., Zorec, J., Frémat, Y. 2006, In Active OB-Stars: laboratories for stellar and circumstellar physics, in press

Andrillat, Y., \& Fehrenbach, Ch. 1982, A\&AS, 48, 93

Ballereau, D., Chauville, J., \& Zorec, J. 1995, A\&ASS, 111, 457

Carciofi, A. C., \& Bjorkman, J. E. 2006, ApJ, 639, 1081

Castor, L., Smith, L. F., \& van Blerkom, D. 1970, ApJ, 159, 1119

Chauville, J., Zorec, J., Ballereau, D., et al. 2001, A\&A 378, 861

Cidale, L. S., \& Ringuelet, A. E. 1989, PASP, 101, 417

Collin-Souffrin, S., Joly, M., Heidmann, N., et al. 1979, A\&A 72, 293

Collin-Souffrin, S., Joly, M., Heidmann, N., et al. 1980, A\&A, 83, 190

Collins II, G. W. 1987, In IAU Coll., 92, 3

Dachs, J., Hanuschik, R. W., Kaiser, D., et al. 1986, A\&A, 159, 276

Dachs, J., Hummel, W., \& Hanuschik, R. W. 1992, A\&ASS, 95, 437

de Geus, E. J., de Zeewv, P. T., \& Lub, J. 1989, A\&A, 216, 44

Divan, L., \& Zorec, J. 1982, ESA-SP, 177, 101

Frémat, Y., Zorec, J., Hubert, A. M., et al. 2005, A\&A, 440, 305

Friedjung, M., \& Malakapur, I. 1971, Astrophys. Lett., 7, 171

Friedjung, M., \& Muratorio, G. 1987, A\&A, 188, 100

Geisel, S. L. 1970, ApJ, 161, 105

Hanuschik, R. W. 1987, A\&A, 173, 299

Hanuschik, R. W. 1988, A\&A, 190, 187
Huang, S. S. 1972, ApJ, 171, 549

Hubert-Delplace, A. M., \& Hubert, H. 1979, An Atlas of Be Stars, Paris Meudon Observatory

Hummel, W. 1994, A\&A, 289, 458

Jaschek, M., Slettebak, M., \& Jaschek, C. 1981, Be Nwsl., 4, 9

Jones, C. E., Sigut, T. A. A., \& Marlborough, J. M. 2004, MNRAS, 352, 841

Kotnik-Karuza, D., Friedjung, M., \& Selvelli, P. L. 2002, A\&A, 381, 507

Kurucz, R., 1992, Models of Stellar Atmospheres, CD Freeman \& Co.

Mihalas, D. 1978, Stellar Atmospheres, 2nd. ed.,

Moujtahid, A., Zorec, J., \& Hubert, A. M. 2000, in IAU Coll., 175, ASP Conf. Ser., 214, 506

Muratorio, G. 1985, Ph.D., Marsella University

Muratorio, G., \& Friedjung, M. 1988, A\&A, 190, 103

Muratorio, G., Viotti, R., Friedjung, M., et al. 1992, A\&A, 258, 423

Muratorio, G., Friedjung, M., Rossi, C., et al. 2002a, Scientific Highlights SF2A 2002, p. 547

Muratorio, G., Markova, N., Friedjung, M., et al. 2002b, A\&A, 390, 213

Neiner, C. 2006, in Active OB-Stars: Laboratories for Stellar and Circumstellar Physics, in press

Neiner, C., Hubert, A. M., \& Frémat, Y., et al. 2003, A\&A, 409, 275

Netzer, H. 1988, in Physics of Formation of Fe II Line Outside LTE, IAU Coll., 94, Ap\&SS Lib. 138, 247

Polidan, R. S., \& Peters, G. J. 1976, Be and Shell Stars, IAU Symp., 70, 59

Selvelli, P., \& Friedjung, M. 2003, A\&A, 401, 297

Slettebak, A. 1982, Be stars, IAU Symp., 98 (Dordrecht: D. Reidel Publishing Co.), 109

Slettebak, A., Collins II, G. W., \& Truax, R. 1992, ApJ, 81, 335

Tarafdar, S. P., \& Apparao, K. M. V. 1994, A\&A, 290, 159

Thomas, R. N. 1965, Non-Equilibrium Thermodynamics in the Presence of a Radiation Field (Univ. Colorado Press)

Thomas, R. N. 1983, Stellar Atmospheric Structural Patterns, NASA SP-471, 76

Vinicius, M. M. F., Zorec, J., Leister, N. V., \& Levenhagen, R. S. 2006, A\&A, 446,643

Viotti, R. 1970, Mem. Soc. Astron. Ital., 41, 513

Viotti, R. 1976, ApJ, 204, 293

Viotti, R., \& Koubský, P. 1976, Be and Shell Stars, ed. A. Slettebak (Dordrecht, Holland; Boston: D. Reidel Pub. Co.), IAU Symp., 70, 99

van den Ancker, M. E., Blondel, P. F. C., Tjin A Djie, H. R. E., et al. 2004, MNRAS, 349, 1516

Wellmann, P. 1952, Z. Astrophys., 30, 71, 88, 96

Yudin, R.V. 2001, A\&A, 368, 912

Zorec, J., Frémat, Y., \& Cidale, L. 2005, A\&A, 441, 235 
M. L. Arias et al.: Fe II emission lines in Be stars, Online Material p 1

\section{Online Material}


M. L. Arias et al.: Fe II emission lines in Be stars, Online Material p 2

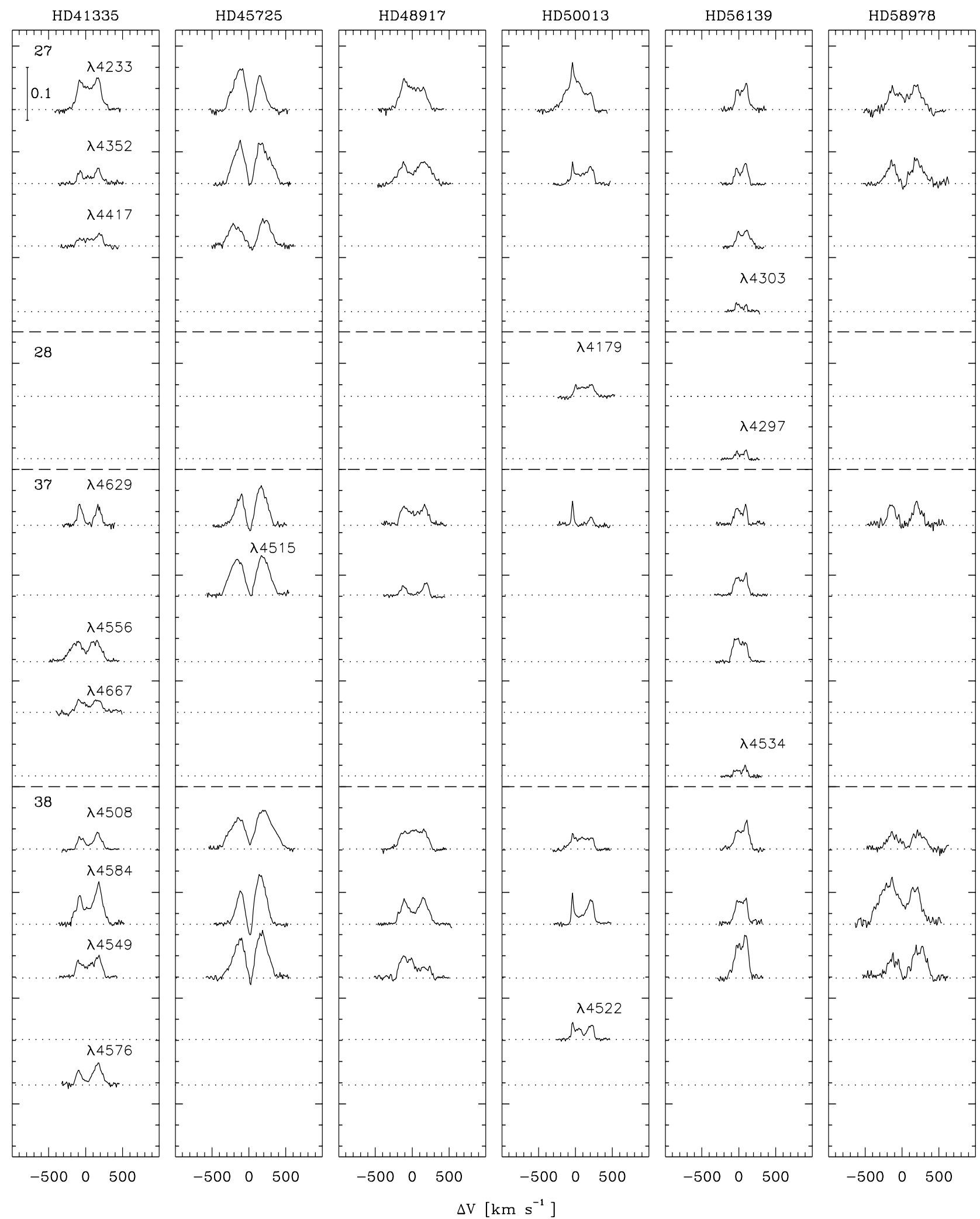

Fig. 7. Fe II and Balmer line-emission profiles of some observed Be stars. The number in the upper left hand corner of each column of Fe II line profiles indicates the line multiplet. 
M. L. Arias et al.: Fe II emission lines in Be stars, Online Material p 3

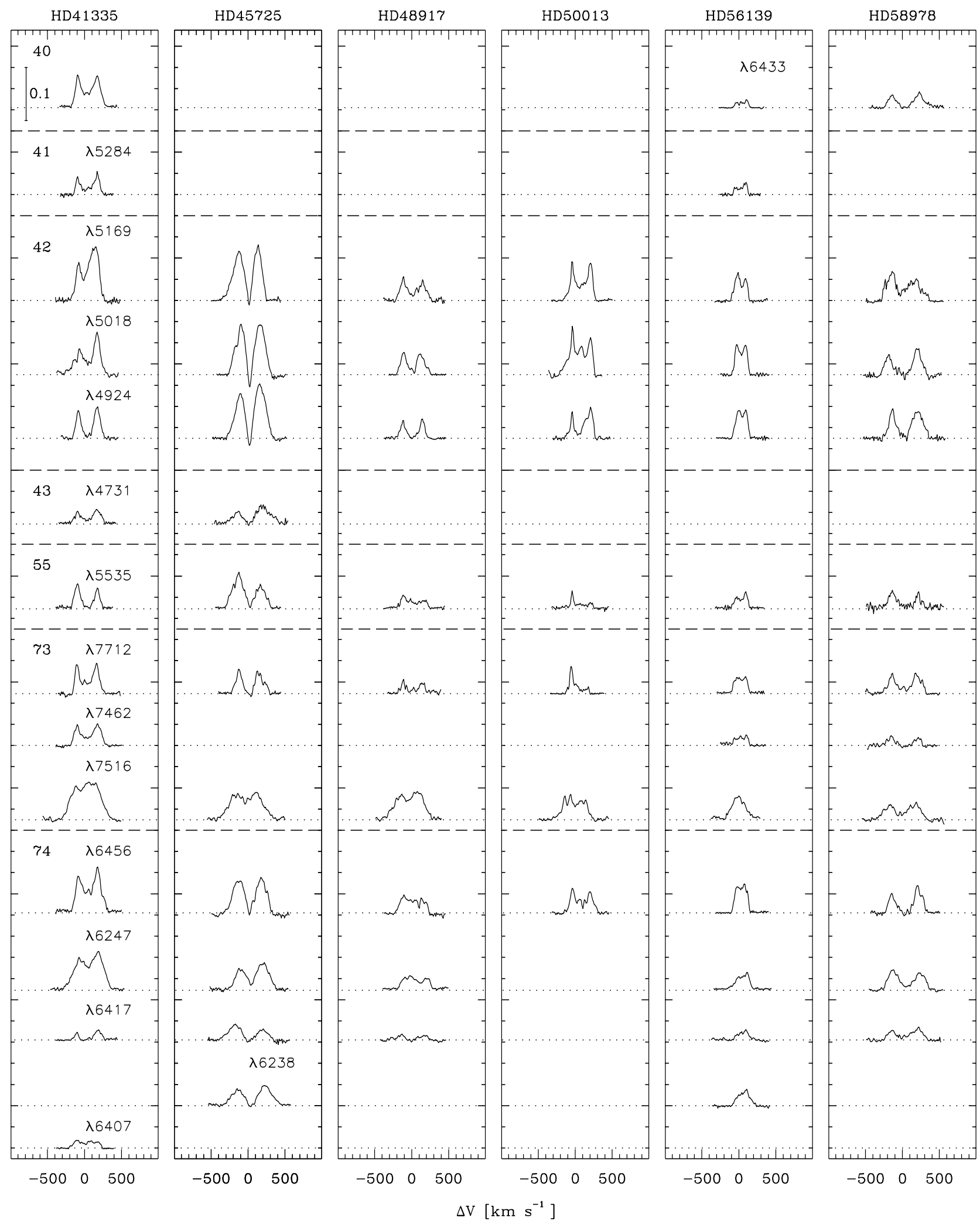

Fig. 8. Same as in Fig. 7. 
M. L. Arias et al.: Fe II emission lines in Be stars, Online Material p 4

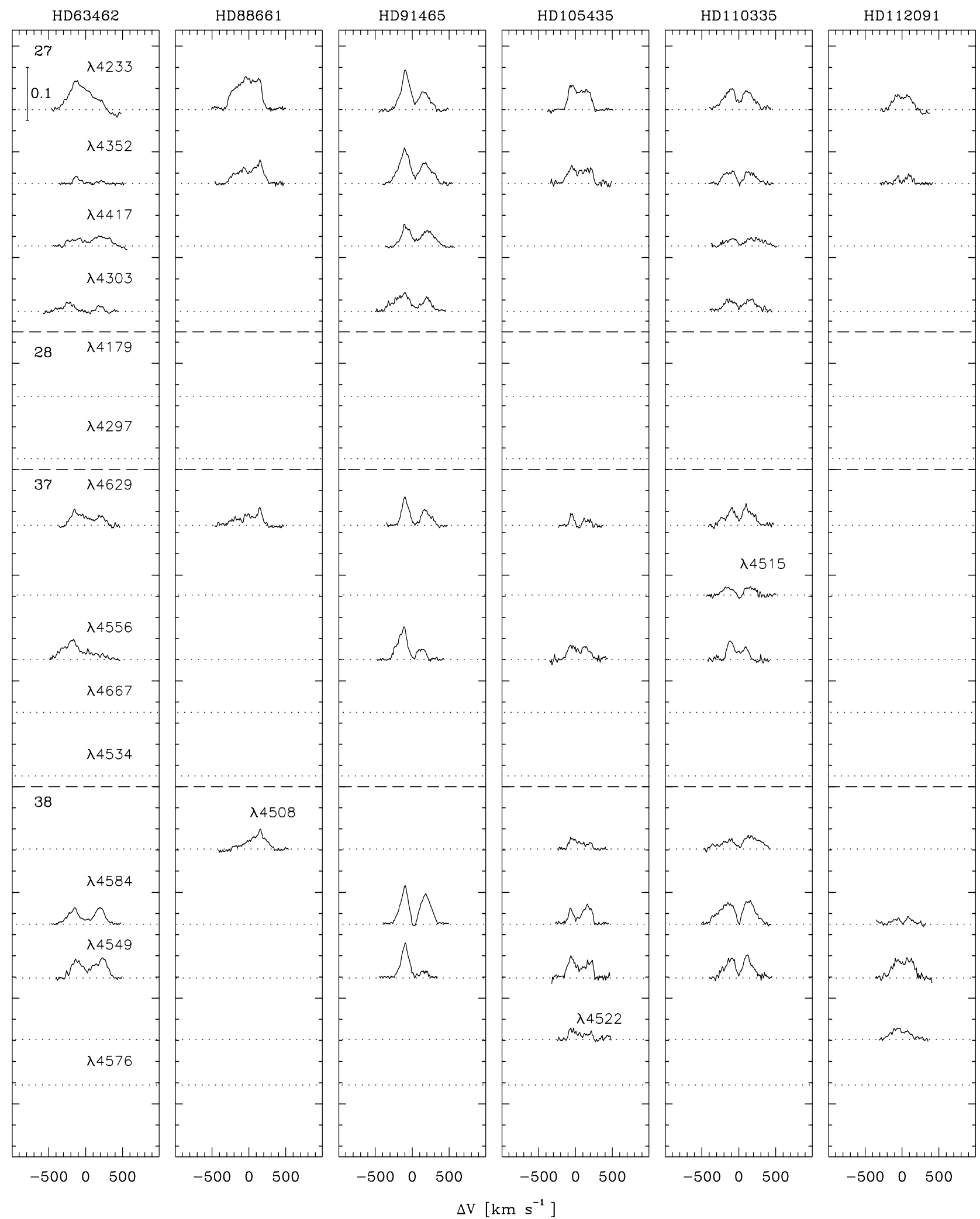

Fig. 9. Same as in Fig. 7. 
M. L. Arias et al.: Fe II emission lines in Be stars, Online Material p 5

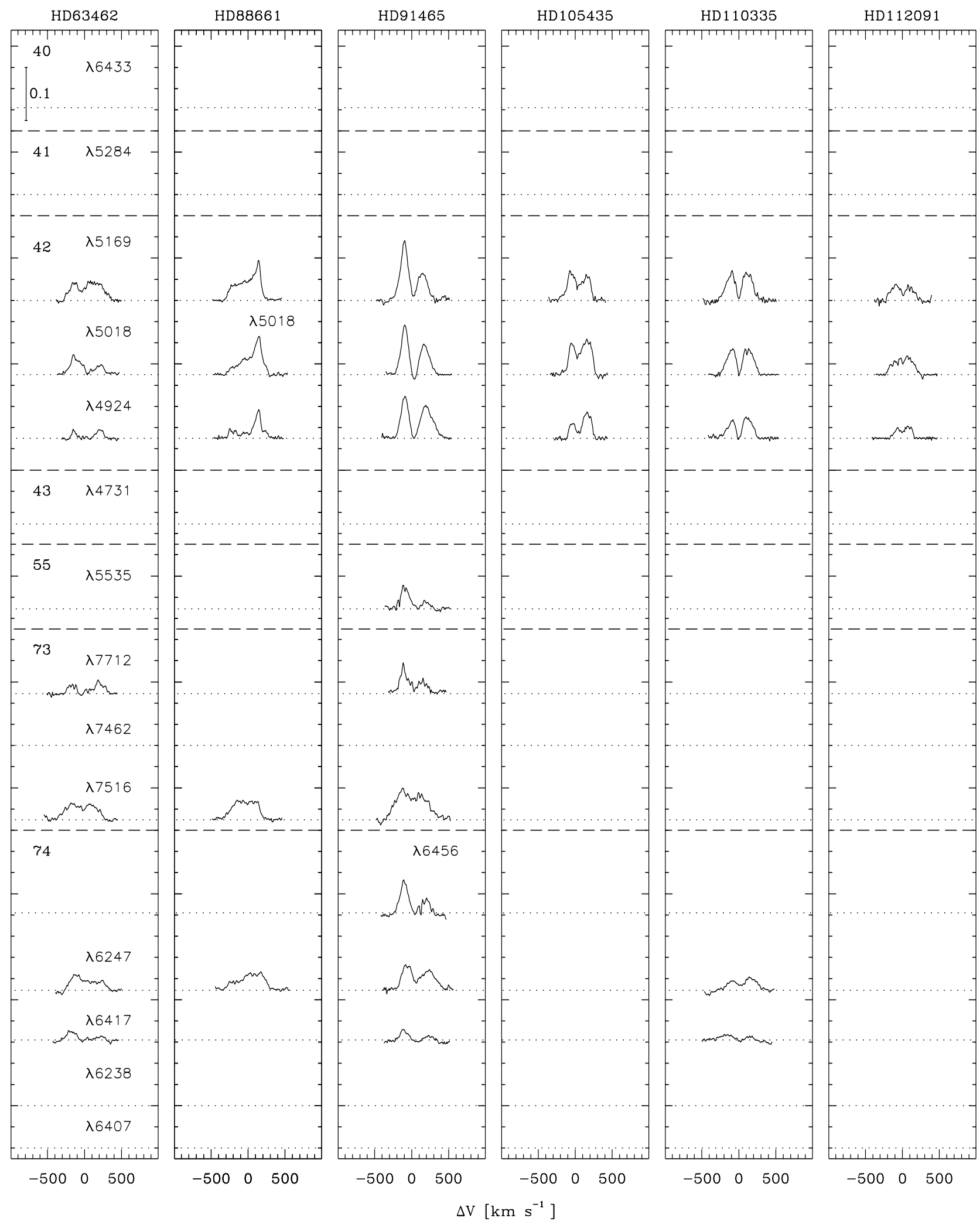

Fig. 10. Same as in Fig. 7. 
M. L. Arias et al.: Fe II emission lines in Be stars, Online Material p 6

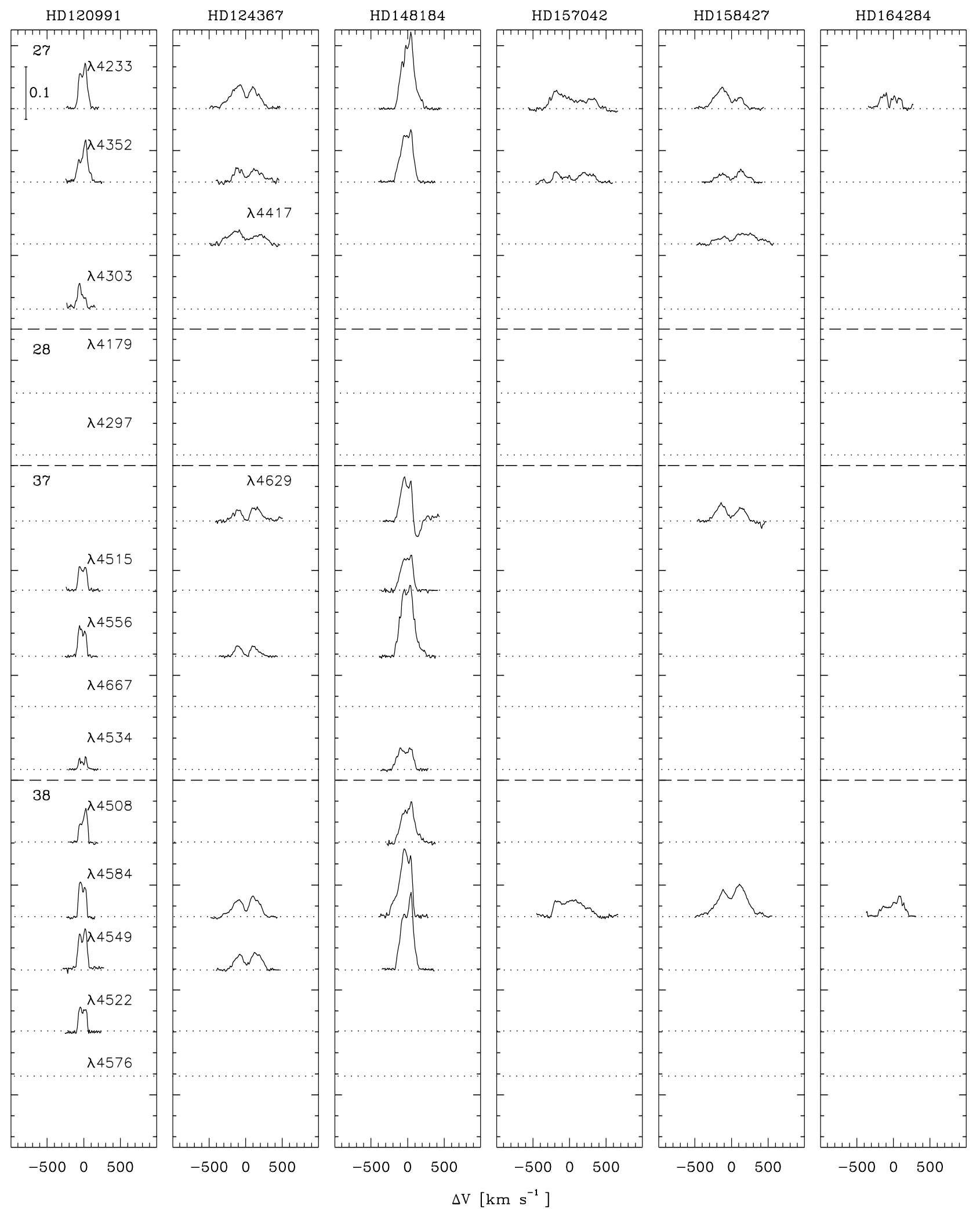

Fig. 11. Same as in Fig. 7. 
M. L. Arias et al.: Fe II emission lines in Be stars, Online Material $p 7$

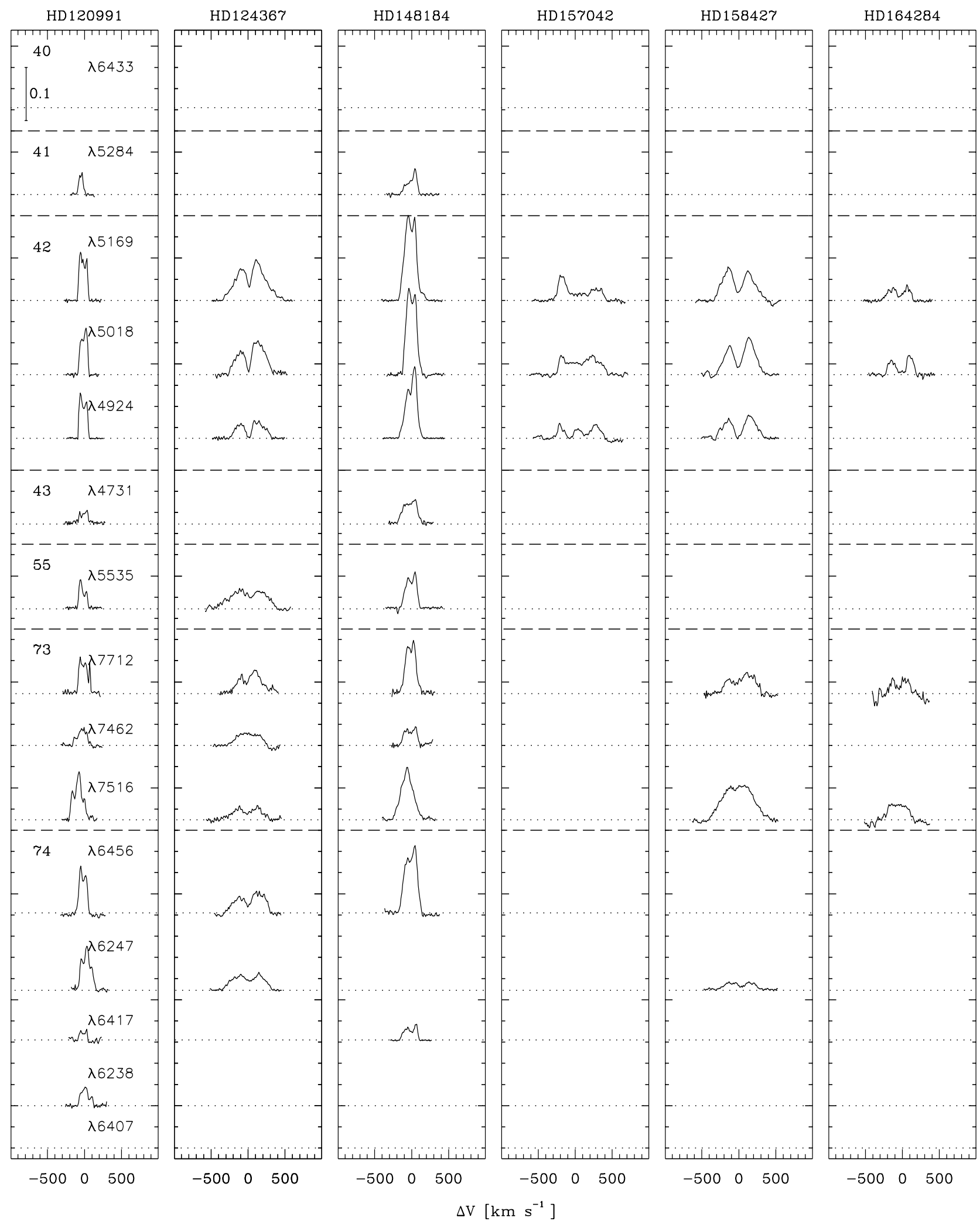

Fig. 12. Same as in Fig. 7. 
M. L. Arias et al.: Fe II emission lines in Be stars, Online Material $p 8$

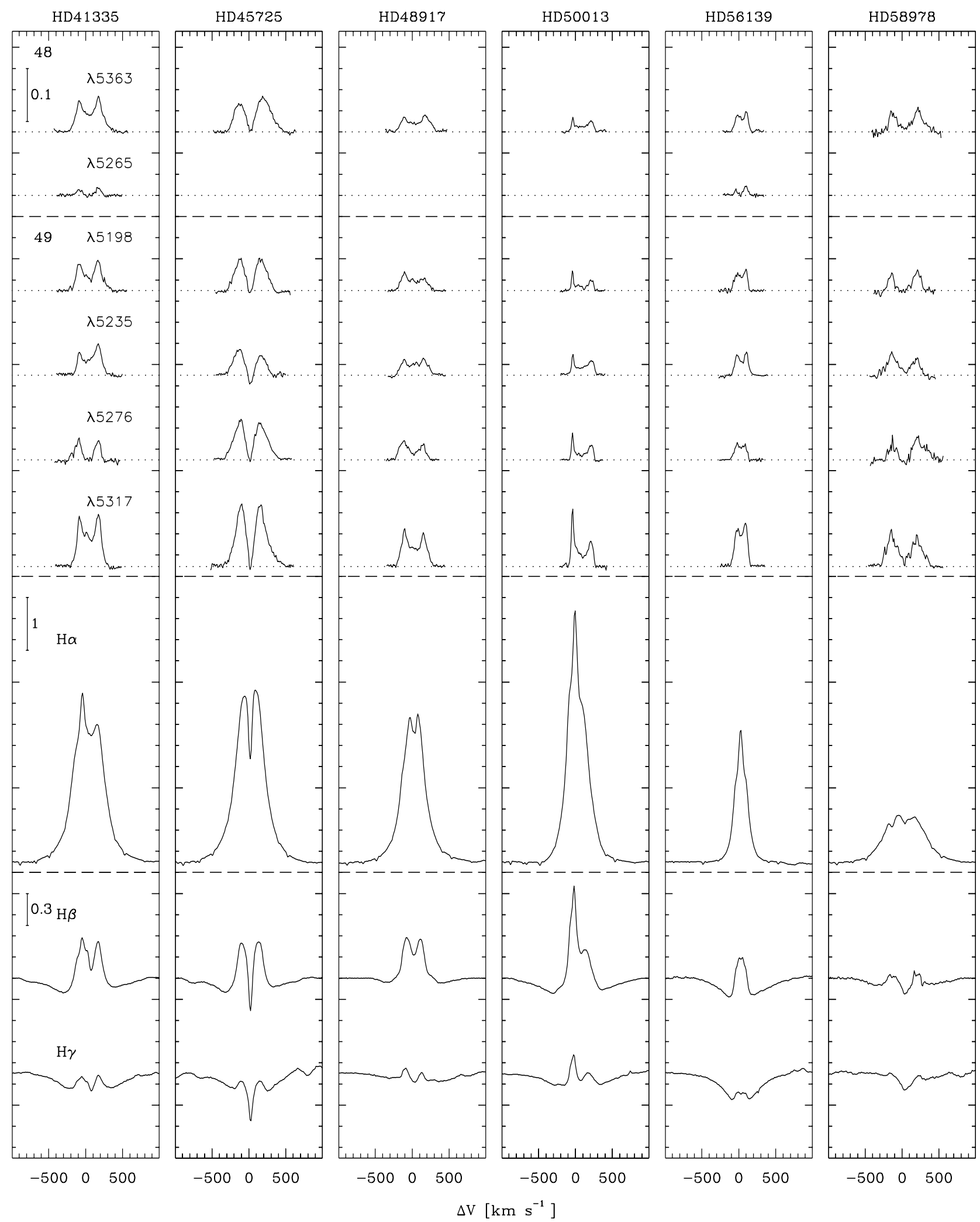

Fig. 13. Fe II and Balmer line emission profiles of some observed Be stars. The number in the upper left hand corner of each column of Fe II line profiles indicates the line multiplet. $\mathrm{H} \beta$ and $\mathrm{H} \gamma$ lines have the same intensity scales, but it is different for $\mathrm{H} \alpha$. 
M. L. Arias et al.: Fe II emission lines in Be stars, Online Material p 9
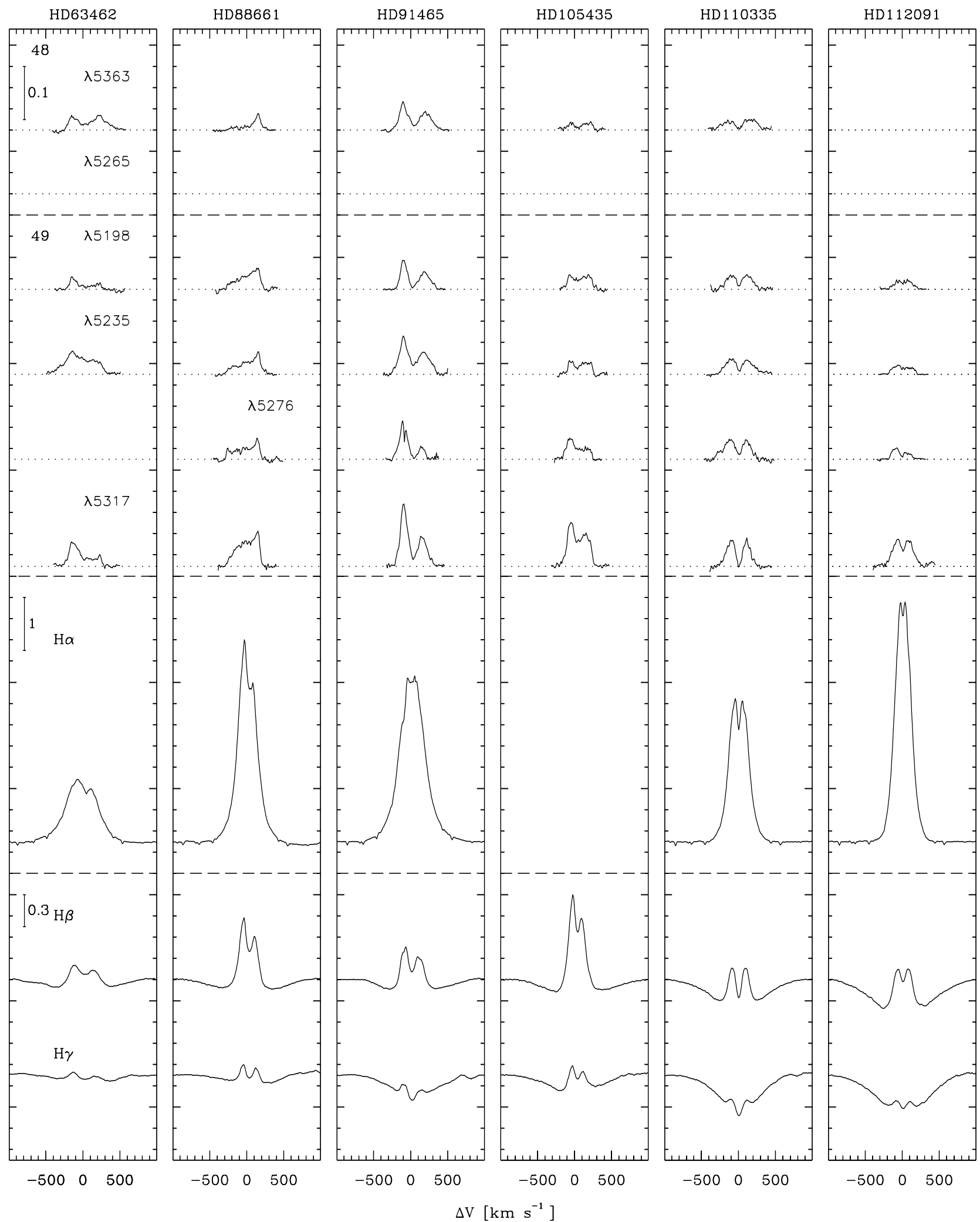

Fig. 14. Same as in Fig. 13. 
M. L. Arias et al.: Fe II emission lines in Be stars, Online Material p 10

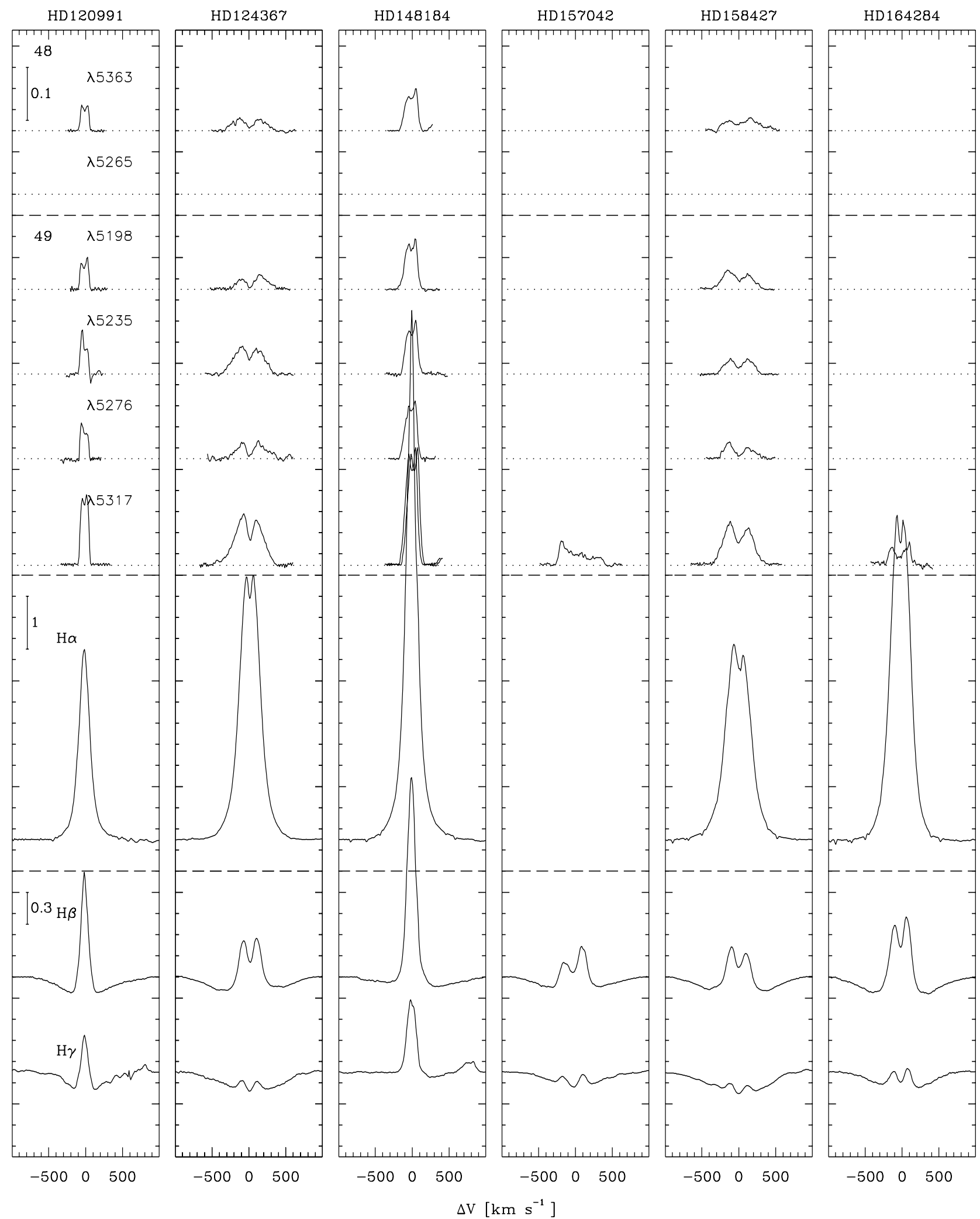

Fig. 15. Same as in Fig. 13. 
M. L. Arias et al.: Fe II emission lines in Be stars, Online Material p 11

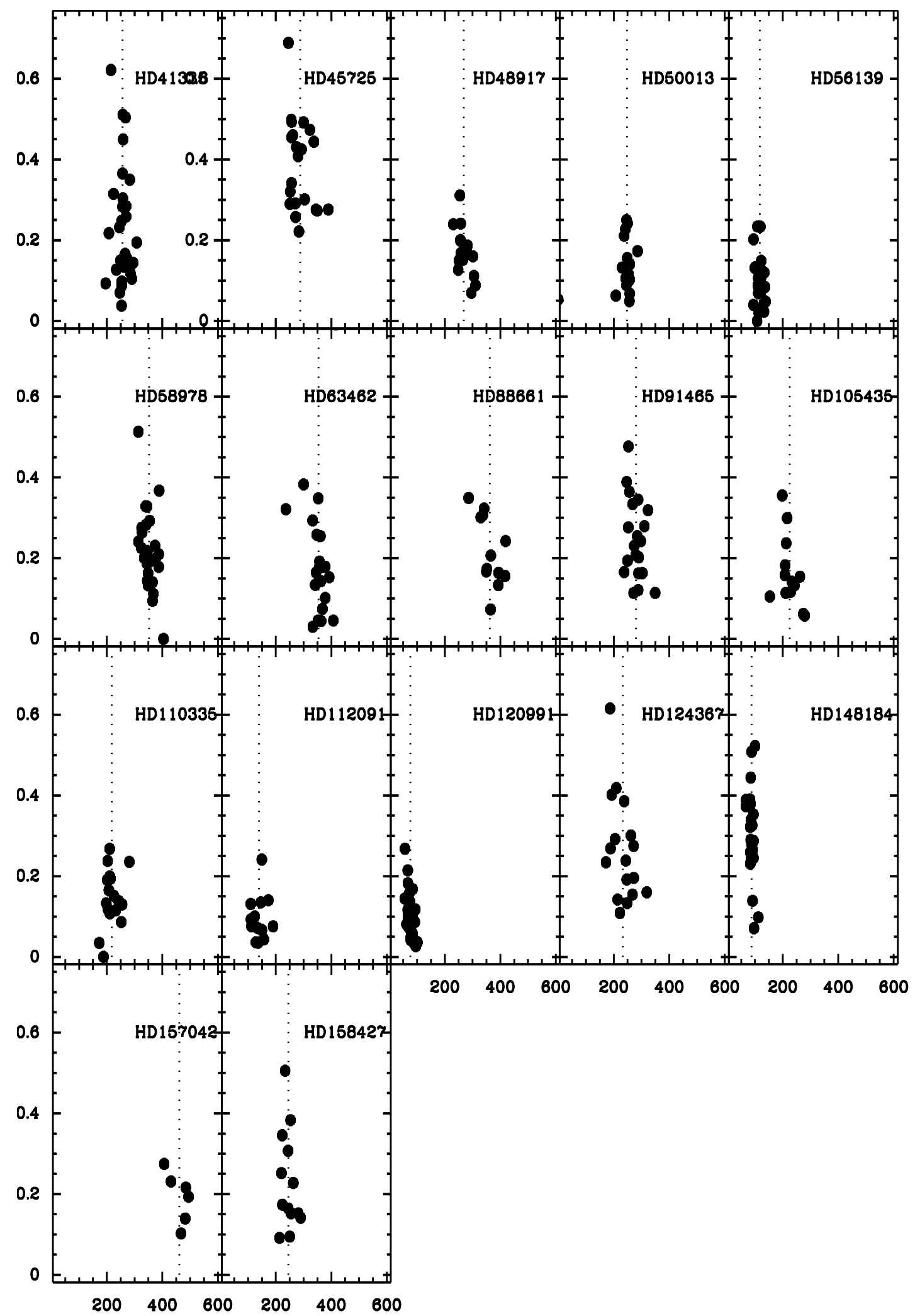

Fig. 16. Equivalent widths $W(\AA)$ of individual Fe II emission lines against their peak separation $\Delta_{\mathrm{p}}\left(\mathrm{km} \mathrm{s}^{-1}\right)$ in all observed Be stars. 
M. L. Arias et al.: Fe II emission lines in Be stars, Online Material $p 12$

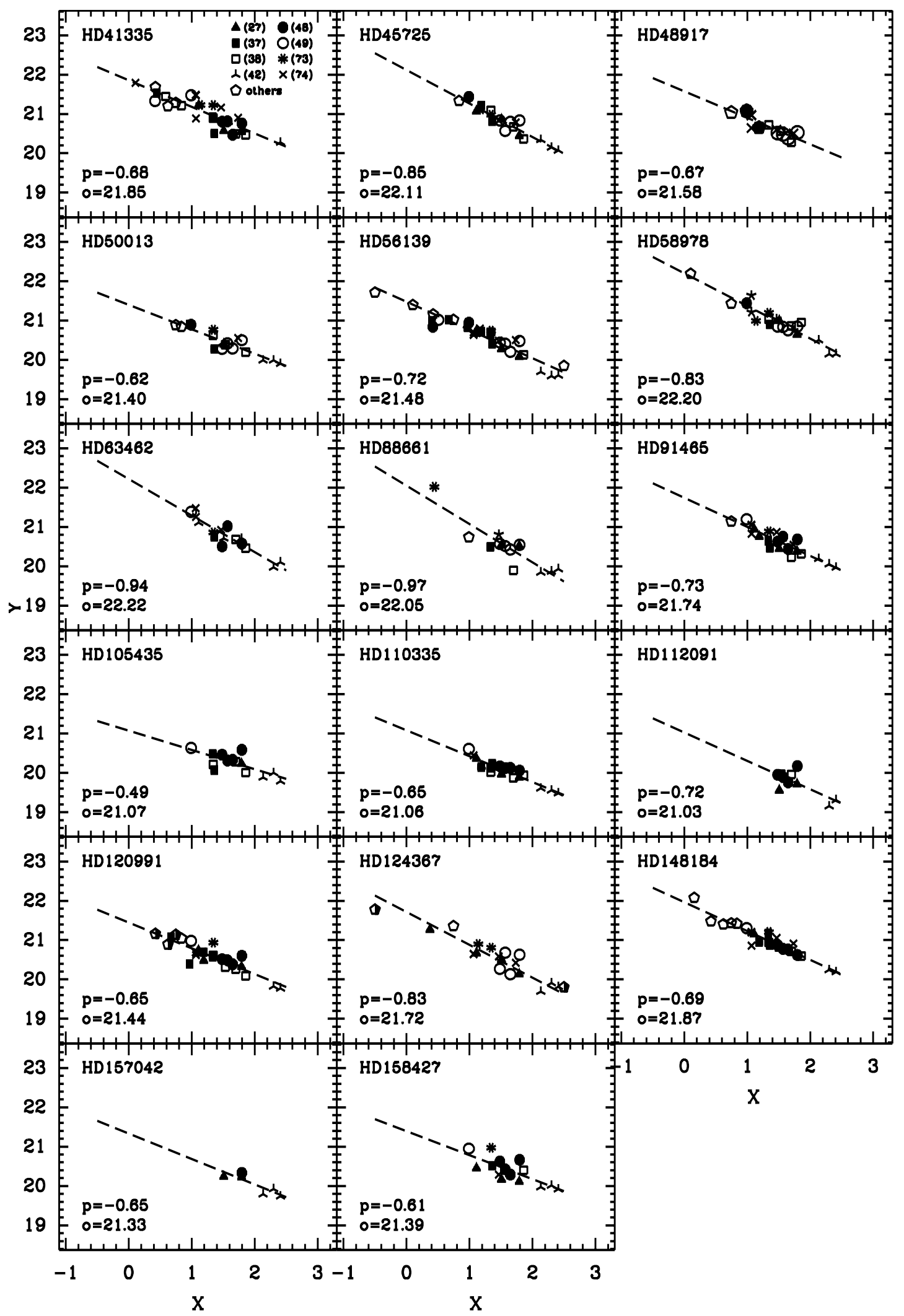

Fig. 17. Empirical SAC slopes for all observed Be stars. Each symbol corresponds to a given multiplet. The correspondence between symbols and multiplets is shown in the first left upper panel. In each panel are also given the slope $p=\partial Y / \partial X$ and $o=$ ordinate at $X=0$. 
M. L. Arias et al.: Fe II emission lines in Be stars, Online Material p 13

Table 4. Journal and spectral ranges of spectroscopic observations.

\begin{tabular}{|c|c|c|c|}
\hline HD & $\begin{array}{c}\text { Date } \\
{[\mathrm{dd} / \mathrm{mm} / \mathrm{yy}]}\end{array}$ & $\begin{array}{c}\text { Spectral Range } \\
\text { [Angstroms] }\end{array}$ & $\begin{array}{c}\text { Julian Date } \\
\text { [days] }\end{array}$ \\
\hline 41335 & $\begin{array}{l}05 / 03 / 96 \\
06 / 03 / 96\end{array}$ & $\begin{array}{l}3900-6450 \\
5600-8100\end{array}$ & $\begin{array}{l}2450148.533 \\
2450149.518\end{array}$ \\
\hline 45725 & 07/03/96 & $\begin{array}{l}3900-6450 \\
5600-8100\end{array}$ & $\begin{array}{l}2450150.506 \\
2450150.666\end{array}$ \\
\hline 47054 & 07/03/96 & $\begin{array}{l}3900-6450 \\
5600-8100\end{array}$ & $\begin{array}{l}2450150.523 \\
2450150.681\end{array}$ \\
\hline 48917 & 07/03/96 & $\begin{array}{l}3900-6450 \\
5600-8100\end{array}$ & $\begin{array}{l}2450150.554 \\
2450150.696\end{array}$ \\
\hline 50013 & $\begin{array}{l}05 / 03 / 96 \\
06 / 03 / 96\end{array}$ & $\begin{array}{l}3900-6450 \\
5600-8100\end{array}$ & $\begin{array}{l}2450148.579 \\
2450149.572\end{array}$ \\
\hline 56139 & 07/03/96 & $\begin{array}{l}3900-6450 \\
5600-8100\end{array}$ & $\begin{array}{l}2450150.609 \\
2450150.732\end{array}$ \\
\hline 58978 & 07/03/96 & $\begin{array}{l}3900-6450 \\
5600-8100\end{array}$ & $\begin{array}{l}2450150.622 \\
2450150.743\end{array}$ \\
\hline 63462 & $\begin{array}{l}05 / 03 / 96 \\
06 / 03 / 96\end{array}$ & $\begin{array}{l}3900-6450 \\
5600-8100\end{array}$ & $\begin{array}{l}2450148.635 \\
2450149.629\end{array}$ \\
\hline 88661 & $\begin{array}{l}05 / 03 / 96 \\
06 / 03 / 96\end{array}$ & $\begin{array}{l}3900-6450 \\
5600-8100\end{array}$ & $\begin{array}{l}2450148.655 \\
2450149.648\end{array}$ \\
\hline 91465 & $\begin{array}{l}05 / 03 / 96 \\
06 / 03 / 96\end{array}$ & $\begin{array}{l}3900-6450 \\
5600-8100\end{array}$ & $\begin{array}{l}2450148.691 \\
2450149.685\end{array}$ \\
\hline 105435 & $\begin{array}{l}05 / 03 / 96 \\
06 / 03 / 96\end{array}$ & $\begin{array}{l}3900-6450 \\
5600-8100\end{array}$ & $\begin{array}{l}2450148.704 \\
2450149.700\end{array}$ \\
\hline 110335 & 07/03/96 & $\begin{array}{l}3900-6450 \\
5600-8100\end{array}$ & $\begin{array}{l}2450150.806 \\
2450150.862\end{array}$ \\
\hline 112091 & 07/03/96 & $\begin{array}{l}3900-6450 \\
5600-8100\end{array}$ & $\begin{array}{l}2450150.821 \\
2450150.891\end{array}$ \\
\hline 120991 & $\begin{array}{l}05 / 03 / 96 \\
06 / 03 / 96\end{array}$ & $\begin{array}{l}3900-6450 \\
5600-8100\end{array}$ & $\begin{array}{l}2450148.877 \\
2450149.876\end{array}$ \\
\hline 124367 & $\begin{array}{l}05 / 03 / 96 \\
06 / 03 / 96\end{array}$ & $\begin{array}{l}3900-6450 \\
5600-8100\end{array}$ & $\begin{array}{l}2450148.777 \\
2450149.769\end{array}$ \\
\hline 148184 & $\begin{array}{l}05 / 03 / 96 \\
06 / 03 / 96 \\
21 / 09 / 96 \\
22 / 09 / 96\end{array}$ & $\begin{array}{l}3900-6450 \\
5600-8100 \\
3700-6150 \\
5850-8100\end{array}$ & $\begin{array}{l}2450148.828 \\
2450149.825 \\
2450348.521 \\
2450349.527\end{array}$ \\
\hline 157042 & 21/09/96 & $3700-6150$ & 2450348.478 \\
\hline 158427 & $\begin{array}{l}21 / 09 / 96 \\
22 / 09 / 96\end{array}$ & $\begin{array}{l}3700-6150 \\
5850-8100\end{array}$ & $\begin{array}{l}2450348.508 \\
2450349.551\end{array}$ \\
\hline 164284 & $\begin{array}{l}21 / 09 / 96 \\
22 / 09 / 96\end{array}$ & $\begin{array}{l}3700-6150 \\
5850-8100\end{array}$ & $\begin{array}{l}2450348.557 \\
2450349.569\end{array}$ \\
\hline
\end{tabular}


M. L. Arias et al.: Fe II emission lines in Be stars, Online Material p 14

Table 5. Measurements of the observed Fe II emission lines.

\begin{tabular}{|c|c|c|c|c|c|c|c|c|}
\hline Multiplet & $\begin{array}{l}\lambda \\
\AA\end{array}$ & $I_{\mathrm{b}}$ & $I_{\mathrm{r}}$ & $I_{\mathrm{cd}}$ & $D_{\mathrm{p}}$ & $\frac{D_{1 / 2}}{\mathrm{~km} \mathrm{~s}^{-1}}$ & $D_{1}$ & $\begin{array}{l}W_{\lambda} \\
\AA\end{array}$ \\
\hline \multicolumn{9}{|c|}{ HD 41335} \\
\hline 27 & 4233.172 & 1.064 & 1.061 & 1.044 & 224 & 350 & 625 & -0.3145 \\
\hline 27 & 4351.769 & - & - & - & - & - & - & -0.1701 \\
\hline 27 & 4416.830 & 1.017 & 1.034 & - & 285 & - & 426 & -0.1185 \\
\hline 37 & 4555.893 & 1.035 & 1.033 & 1.011 & 207 & 356 & 429 & -0.2175 \\
\hline 37 & 4629.339 & 1.038 & 1.038 & 1.000 & 253 & - & - & -0.0979 \\
\hline 37 & 4666.758 & 1.018 & 1.031 & 1.010 & 234 & - & 435 & -0.1271 \\
\hline 38 & 4508.288 & 1.047 & 1.050 & 1.029 & 245 & 385 & 457 & -0.2321 \\
\hline 38 & 4549.474 & 1.032 & 1.059 & 1.019 & 282 & 435 & 541 & -0.3498 \\
\hline 38 & 4576.340 & 1.031 & 1.039 & 1.013 & 249 & 362 & 436 & -0.1499 \\
\hline 38 & 4583.837 & 1.055 & 1.078 & 1.027 & 256 & 350 & 545 & -0.2830 \\
\hline 40 & 6432.628 & 1.066 & 1.064 & 1.031 & 220 & 363 & 494 & -0.3314 \\
\hline 41 & 5284.109 & 1.040 & 1.047 & 1.015 & 265 & 334 & 436 & -0.1603 \\
\hline 42 & 4923.927 & 1.052 & 1.057 & 1.003 & 245 & 338 & 440 & -0.1733 \\
\hline 42 & 5018.440 & 1.048 & 1.079 & 1.022 & 259 & 315 & 591 & -0.2812 \\
\hline 42 & 5169.033 & 1.101 & 1.120 & 1.067 & 214 & 346 & 472 & -0.6215 \\
\hline 43 & 4731.453 & 1.023 & 1.025 & 1.005 & 254 & 357 & 452 & -0.0880 \\
\hline 48 & 5264.812 & 1.013 & 1.022 & 1.002 & 246 & 347 & 440 & -0.0702 \\
\hline 48 & 5362.869 & 1.055 & 1.072 & 1.032 & 256 & 390 & 546 & -0.3652 \\
\hline 49 & 5197.577 & 1.049 & 1.057 & 1.023 & 252 & 354 & 427 & -0.2477 \\
\hline 49 & 5234.625 & 1.049 & 1.068 & 1.033 & 257 & 359 & 475 & -0.3039 \\
\hline 49 & 5276.002 & 1.045 & 1.045 & 1.005 & 266 & 361 & 421 & -0.1659 \\
\hline 49 & 5316.615 & 1.094 & 1.097 & 1.046 & 258 & 334 & 461 & -0.4496 \\
\hline 55 & 5534.847 & 1.045 & 1.037 & 1.002 & 265 & 350 & 423 & -0.1339 \\
\hline 73 & 7462.407 & 1.039 & 1.041 & 1.016 & 269 & 388 & 465 & -0.2586 \\
\hline 73 & 7515.831 & 1.072 & 0 & 0 & 268 & 477 & 846 & -0.7788 \\
\hline 73 & 7711.723 & 1.052 & 1.053 & 1.019 & 269 & 338 & 423 & -0.2844 \\
\hline 74 & 6147.741 & - & - & - & - & - & - & -0.2991 \\
\hline 74 & 6149.258 & - & - & - & - & - & - & -0.2991 \\
\hline 74 & 6247.557 & 1.059 & 1.071 & 1.040 & 267 & 417 & 574 & -0.5041 \\
\hline 74 & 6407.251 & 1.015 & 1.013 & 1.007 & 195 & - & 457 & -0.0930 \\
\hline 74 & 6416.919 & 1.019 & 1.023 & 1.004 & 289 & 385 & 506 & -0.1047 \\
\hline 74 & 6456.383 & 1.073 & 1.090 & 1.038 & 257 & 349 & 472 & -0.5106 \\
\hline \multicolumn{9}{|c|}{ HD 45725} \\
\hline 27 & 4233.172 & 1.075 & 1.063 & 1.000 & 251 & 443 & 653 & -0.3204 \\
\hline 27 & 4351.769 & 1.082 & 1.074 & 1.000 & 273 & 523 & 721 & -0.4304 \\
\hline 27 & 4416.830 & 1.041 & 1.056 & 1.000 & 389 & 607 & 761 & -0.2760 \\
\hline 37 & 4515.339 & 1.069 & 1.074 & 1.000 & 337 & 589 & 735 & -0.4438 \\
\hline 37 & 4629.339 & 1.049 & 1.064 & 1.000 & 271 & 433 & 581 & -0.2573 \\
\hline 38 & 4508.288 & 1.062 & 1.077 & 1.013 & 322 & 668 & 884 & -0.4735 \\
\hline 38 & 4549.474 & 1.070 & 1.082 & 0.990 & 280 & 502 & 694 & -0.4081 \\
\hline 38 & 4583.837 & 1.062 & 1.086 & 0.980 & 270 & 422 & 560 & -0.2913 \\
\hline 42 & 4923.927 & 1.084 & 1.102 & 0.990 & 256 & 434 & 642 & -0.4931 \\
\hline 42 & 5018.440 & 1.095 & 1.094 & 0.980 & 261 & 435 & 595 & -0.4600 \\
\hline 42 & 5169.033 & 1.092 & 1.103 & 0.990 & 255 & 395 & 549 & -0.4982 \\
\hline 43 & 4731.453 & 1.020 & 1.033 & 0.994 & 334 & 514 & 716 & -0.1500 \\
\hline 48 & 5362.869 & 1.051 & 1.065 & 1.002 & 292 & 535 & 748 & -0.4249 \\
\hline 49 & 5197.577 & 1.059 & 1.060 & 0.990 & 256 & 466 & 643 & -0.3414 \\
\hline 49 & 5234.625 & 1.049 & 1.036 & 0.990 & 283 & 469 & 606 & -0.2214 \\
\hline 49 & 5276.002 & 1.076 & 1.070 & 1.000 & 256 & 478 & 748 & -0.4551 \\
\hline 49 & 5316.615 & 1.117 & 1.112 & 0.990 & 245 & 429 & 641 & -0.6889 \\
\hline 55 & 5534.847 & 1.028 & 1.067 & 1.000 & 292 & 464 & 620 & -0.3299 \\
\hline 73 & 7515.831 & 1.050 & 1.051 & 0 & 250 & 474 & 799 & -0.5811 \\
\hline 73 & 7711.723 & 1.047 & 1.045 & 1.000 & 250 & 360 & 525 & -0.2900 \\
\hline 74 & 6238.392 & 1.030 & 1.037 & 1.000 & 349 & 577 & 790 & -0.2733 \\
\hline 74 & 6247.557 & 1.033 & 1.047 & 1.000 & 303 & 501 & 622 & -0.3013 \\
\hline 74 & 6416.919 & 1.034 & 1.024 & 1.000 & 367 & 571 & 776 & -0.2524 \\
\hline 74 & 6456.383 & 1.062 & 1.069 & 1.000 & 300 & 498 & 632 & -0.4919 \\
\hline
\end{tabular}


M. L. Arias et al.: Fe II emission lines in Be stars, Online Material p 15

Table 5. continued.

\begin{tabular}{|c|c|c|c|c|c|c|c|c|}
\hline Multiplet & $\begin{array}{l}\lambda \\
\AA\end{array}$ & $I_{\mathrm{b}}$ & $I_{\mathrm{r}}$ & $I_{\mathrm{cd}}$ & $D_{\mathrm{p}}$ & $\begin{array}{l}D_{1 / 2} \\
\mathrm{~km} \mathrm{~s}^{-1}\end{array}$ & $D_{1}$ & $\begin{array}{c}W_{\lambda} \\
\AA\end{array}$ \\
\hline \multicolumn{9}{|c|}{ HD 48917} \\
\hline 27 & 4233.172 & 1.059 & 1.042 & - & 257 & 365 & 503 & -0.2410 \\
\hline 27 & 4351.769 & 1.041 & 1.041 & 1.018 & 269 & 458 & 681 & -0.2268 \\
\hline 37 & 4515.339 & 1.021 & 1.035 & 1.006 & 304 & 424 & 461 & -0.1113 \\
\hline 37 & 4629.339 & 1.030 & 1.034 & 1.014 & 283 & 377 & 455 & -0.1342 \\
\hline 38 & 4508.288 & 1.028 & 1.036 & - & 281 & 413 & 483 & -0.1868 \\
\hline 38 & 4549.474 & 1.041 & 1.014 & - & 264 & - & 487 & -0.1511 \\
\hline 38 & 4583.837 & 1.052 & 1.041 & 1.014 & 256 & 403 & 495 & -0.1985 \\
\hline 42 & 4923.927 & 1.033 & 1.036 & 0.999 & 253 & 331 & 457 & -0.0974 \\
\hline 42 & 5018.440 & 1.041 & 1.038 & 1.005 & 213 & 334 & 466 & -0.1587 \\
\hline 42 & 5169.033 & 1.044 & 1.039 & 1.008 & 260 & 346 & 489 & -0.1679 \\
\hline 48 & 5362.869 & 1.028 & 1.032 & 1.013 & 278 & 428 & 521 & -0.1710 \\
\hline 49 & 5197.577 & 1.036 & 1.020 & - & 250 & 377 & 484 & -0.1497 \\
\hline 49 & 5234.625 & 1.030 & 1.032 & - & 257 & 401 & 507 & -0.1678 \\
\hline 49 & 5276.002 & 1.036 & 1.031 & 1.011 & 265 & 368 & 472 & -0.1601 \\
\hline 49 & 5316.615 & 1.069 & 1.063 & 1.031 & 254 & 358 & 498 & -0.3108 \\
\hline 55 & 5534.847 & 1.024 & 1.015 & 1.006 & 310 & 360 & 412 & -0.0883 \\
\hline 73 & 7515.831 & 1.048 & 1.052 & 1.036 & 210 & 419 & 608 & -0.5070 \\
\hline 73 & 7711.723 & 1.029 & 1.022 & 0 & 243 & 331 & 399 & -0.1185 \\
\hline 74 & 6147.741 & - & - & - & - & - & - & -0.1504 \\
\hline 74 & 6149.258 & - & - & - & - & - & - & -0.1504 \\
\hline 74 & 6247.557 & 1.023 & 1.020 & 1.012 & 301 & 415 & 474 & -0.1601 \\
\hline 74 & 6416.919 & 1.013 & 1.011 & 1.000 & 295 & - & 532 & -0.0698 \\
\hline 74 & 6456.383 & 1.038 & 1.033 & - & 230 & 391 & 477 & -0.2396 \\
\hline \multicolumn{9}{|c|}{ HD 50013} \\
\hline 27 & 4233.172 & 1.089 & 1.032 & - & 246 & 362 & 440 & -0.2497 \\
\hline 27 & 4351.769 & 1.041 & 1.032 & - & 242 & 310 & 360 & -0.1056 \\
\hline 28 & 4178.862 & 1.020 & 1.019 & - & 207 & 269 & 306 & -0.0626 \\
\hline 37 & 4629.339 & - & - & - & - & - & 360 & -0.0533 \\
\hline 38 & 4508.288 & 1.031 & 1.022 & - & 253 & 332 & 410 & -0.1154 \\
\hline 38 & 4522.634 & 1.033 & 1.028 & - & 257 & 306 & 359 & -0.1029 \\
\hline 38 & 4583.837 & 1.059 & 1.043 & 1.013 & 257 & 306 & 375 & -0.1407 \\
\hline 42 & 4923.927 & 1.049 & 1.059 & 1.008 & 248 & 312 & 365 & -0.1559 \\
\hline 42 & 5018.440 & - & - & - & - & - & - & -0.2293 \\
\hline 42 & 5169.033 & 1.073 & 1.071 & 1.024 & 249 & 305 & 379 & -0.2419 \\
\hline 48 & 5362.869 & 1.030 & 1.022 & 1.011 & 245 & 304 & 377 & -0.0884 \\
\hline 49 & 5197.577 & 1.035 & 1.018 & 1.007 & 257 & 306 & 358 & -0.0675 \\
\hline 49 & 5234.625 & 1.039 & 1.027 & 1.014 & 247 & 312 & 373 & -0.1123 \\
\hline 49 & 5276.002 & 1.051 & 1.027 & 1.008 & 255 & 298 & 348 & -0.1006 \\
\hline 49 & 5316.615 & 1.110 & 1.049 & 1.022 & 242 & 292 & 375 & -0.2278 \\
\hline 55 & 5534.847 & 1.032 & 1.009 & 1.004 & 256 & 268 & 336 & -0.0492 \\
\hline 73 & 7515.831 & 1.044 & 1.035 & 0 & 216 & 353 & 570 & -0.3399 \\
\hline 73 & 7711.723 & 1.052 & 1.015 & - & 229 & - & 338 & -0.1323 \\
\hline 74 & 6456.383 & 1.046 & 1.040 & - & 237 & 323 & 429 & -0.2110 \\
\hline \multicolumn{9}{|c|}{ HD 56139} \\
\hline 27 & 4233.172 & 1.037 & 1.049 & - & 119 & 181 & 279 & -0.1071 \\
\hline 27 & 4303.176 & 1.015 & 1.010 & - & 113 & 166 & 207 & -0.0204 \\
\hline 27 & 4351.769 & - & - & - & 122 & 184 & 262 & -0.0856 \\
\hline 27 & 4416.830 & 1.029 & 1.031 & 1.022 & 111 & 188 & 290 & -0.0844 \\
\hline 28 & 4296.572 & 1.014 & 1.015 & 1.004 & 119 & 179 & 225 & -0.0277 \\
\hline 37 & 4515.339 & 1.034 & 1.044 & - & 122 & 207 & 289 & -0.1086 \\
\hline 37 & 4534.168 & 1.011 & 1.021 & 1.007 & 129 & 199 & 232 & -0.0337 \\
\hline 37 & 4555.893 & 1.042 & 1.037 & 1.032 & 119 & 223 & 278 & -0.1303 \\
\hline 37 & 4629.339 & 1.029 & 1.038 & 1.015 & 118 & 177 & 232 & -0.0783 \\
\hline 38 & 4508.288 & 1.038 & 1.057 & 1.032 & 122 & 203 & 332 & -0.1489 \\
\hline 38 & 4549.474 & 1.064 & 1.081 & 1.055 & 119 & 204 & 306 & -0.2335 \\
\hline 38 & 4583.837 & 1.044 & 1.045 & - & 113 & 181 & 281 & -0.1294 \\
\hline 40 & 6432.628 & 1.009 & 1.015 & 1.008 & 131 & 189 & 239 & -0.0419 \\
\hline 41 & 5284.109 & 1.011 & 1.023 & 1.010 & 138 & 191 & 218 & -0.0481 \\
\hline 42 & 4923.927 & 1.020 & 1.027 & 1.007 & 94 & 139 & 171 & -0.0399 \\
\hline 42 & 5018.440 & 1.079 & 1.046 & 1.034 & 118 & 181 & 232 & -0.9890 \\
\hline 42 & 5169.033 & 1.053 & 1.041 & 1.023 & 99 & 186 & 253 & -0.1313 \\
\hline
\end{tabular}


M. L. Arias et al.: Fe II emission lines in Be stars, Online Material p 16

Table 5. continued.

\begin{tabular}{|c|c|c|c|c|c|c|c|c|}
\hline Multiplet & $\begin{array}{l}\lambda \\
\AA\end{array}$ & $I_{\mathrm{b}}$ & $I_{\mathrm{r}}$ & $I_{\mathrm{cd}}$ & $D_{\mathrm{p}}$ & $\frac{D_{1 / 2}}{\mathrm{~km} \mathrm{~s}^{-1}}$ & $D_{1}$ & $\begin{array}{c}W_{\lambda} \\
\AA\end{array}$ \\
\hline 48 & 5264.812 & 1.013 & 1.017 & 0.997 & 132 & 179 & 234 & -0.0229 \\
\hline 48 & 5362.869 & 1.032 & 1.038 & 1.024 & 118 & 187 & 281 & -0.1081 \\
\hline 49 & 5197.577 & 1.034 & 1.041 & 1.024 & 112 & 182 & 250 & -0.1060 \\
\hline 49 & 5234.625 & 1.039 & 1.044 & 1.025 & 133 & 188 & 261 & -0.1198 \\
\hline 49 & 5276.002 & 1.033 & 1.031 & 1.021 & 110 & 172 & 262 & -0.0889 \\
\hline 49 & 5316.615 & 1.069 & 1.080 & 1.056 & 108 & 189 & 273 & -0.2340 \\
\hline 55 & 5534.847 & 1.021 & 1.030 & 1.013 & 121 & 186 & 246 & -0.0721 \\
\hline 73 & 7462.407 & - & 1.019 & - & 114 & 209 & 243 & -0.0767 \\
\hline 73 & 7515.831 & 0 & 0 & 0 & 84 & 229 & 389 & -0.2354 \\
\hline 73 & 7711.723 & 1.025 & 1.030 & 1.022 & 100 & 197 & 238 & -0.1329 \\
\hline 74 & 6147.741 & - & - & - & - & - & - & -0.0601 \\
\hline 74 & 6149.258 & - & - & - & - & - & - & -0.0601 \\
\hline 74 & 6238.392 & 1.020 & 1.031 & - & 111 & 201 & 324 & -0.1068 \\
\hline 74 & 6247.557 & 1.023 & 1.031 & 1.021 & 112 & 200 & 272 & -0.1063 \\
\hline 74 & 6416.919 & 1.014 & 1.022 & - & 111 & 195 & 247 & -0.0688 \\
\hline 74 & 6456.383 & 1.049 & 1.054 & 1.046 & 94 & 189 & 266 & -0.2021 \\
\hline \multicolumn{9}{|c|}{ HD 58978} \\
\hline 27 & 4233.172 & 1.046 & 1.043 & 1.021 & 324 & 479 & 606 & -0.2249 \\
\hline 27 & 4351.769 & 1.041 & 1.042 & 1.000 & 327 & 475 & 708 & -0.2637 \\
\hline 37 & 4629.339 & 1.032 & 1.039 & 1.000 & 345 & 435 & 536 & -0.1439 \\
\hline 38 & 4508.288 & 1.035 & 1.036 & 1.005 & 344 & 471 & 573 & -0.1866 \\
\hline 38 & 4549.474 & 1.047 & 1.047 & 1.000 & 354 & 559 & 589 & -0.2925 \\
\hline 38 & 4583.837 & 1.088 & 1.061 & 1.034 & 313 & 544 & 666 & -0.5131 \\
\hline 40 & 6432.628 & 1.024 & 1.030 & 1.001 & 364 & 477 & 546 & -0.1404 \\
\hline 42 & 4923.927 & 1.053 & 1.066 & 1.005 & 340 & 527 & 748 & -0.3284 \\
\hline 42 & 5018.440 & 1.028 & 1.060 & 1.002 & 386 & - & 608 & -0.2098 \\
\hline 42 & 5169.033 & 1.055 & 1.040 & 1.008 & 325 & 475 & 526 & -0.2753 \\
\hline 48 & 5362.869 & 1.036 & 1.047 & 1.006 & 334 & 463 & 499 & -0.2006 \\
\hline 49 & 5197.577 & 1.031 & 1.038 & 1.000 & 348 & 455 & 548 & -0.1628 \\
\hline 49 & 5234.625 & 1.044 & 1.032 & 1.005 & 344 & 450 & 583 & -0.1918 \\
\hline 49 & 5276.002 & 1.029 & 1.040 & 1.000 & 352 & 550 & 610 & -0.1903 \\
\hline 49 & 5316.615 & 1.067 & 1.057 & 1.001 & 345 & 467 & 582 & -0.3281 \\
\hline 55 & 5534.847 & 1.032 & 1.030 & 1.000 & 367 & 427 & 465 & -0.1117 \\
\hline 73 & 7462.407 & 1.018 & 1.015 & 1.000 & 364 & 484 & 572 & -0.0943 \\
\hline 73 & 7515.831 & 1.028 & 1.030 & 1.007 & 341 & 528 & 680 & -0.2829 \\
\hline 73 & 7711.723 & 1.036 & 1.036 & 1.002 & 313 & 426 & 580 & -0.2408 \\
\hline 74 & 6149.258 & 1.027 & 1.052 & 1.008 & 388 & - & 684 & -0.3674 \\
\hline 74 & 6247.557 & 1.035 & 1.030 & 1.004 & 373 & 539 & 611 & -0.2305 \\
\hline 74 & 6416.919 & 1.018 & 1.024 & 1.006 & 357 & 475 & 591 & -0.1377 \\
\hline 74 & 6456.383 & 1.037 & 1.051 & 1.000 & 346 & 479 & 580 & -0.2174 \\
\hline \multicolumn{9}{|c|}{ HD 63462} \\
\hline 27 & 4233.172 & 1.053 & 1.017 & - & 347 & 430 & 684 & -0.2581 \\
\hline 27 & 4303.176 & 1.016 & 1.007 & 0 & 407 & 0 & 585 & -0.0454 \\
\hline 27 & 4351.769 & 1.013 & 1.007 & - & 332 & - & 480 & -0.0299 \\
\hline 27 & 4416.830 & 1.016 & 1.020 & 1.008 & 341 & - & 721 & -0.1339 \\
\hline 37 & 4555.893 & 1.037 & 1.012 & 0 & 403 & 0 & 640 & -0.1738 \\
\hline 37 & 4629.339 & 1.031 & 1.015 & 0 & 351 & 478 & 581 & -0.1281 \\
\hline 38 & 4549.474 & 1.032 & 1.036 & 1.011 & 357 & 502 & 606 & -0.1915 \\
\hline 38 & 4583.837 & 1.031 & 1.031 & 1.007 & 344 & 506 & 723 & -0.1645 \\
\hline 42 & 4923.927 & 1.017 & 1.015 & 0.999 & 363 & 434 & 479 & -0.0444 \\
\hline 42 & 5018.440 & 1.038 & 1.017 & 1.003 & 362 & 460 & 524 & -0.1429 \\
\hline 42 & 5169.033 & 1.034 & 1.035 & 1.018 & 284 & 501 & 649 & -0.2583 \\
\hline 48 & 5362.869 & 1.025 & 1.028 & 1.011 & 378 & 503 & 622 & -0.1788 \\
\hline 49 & 5197.577 & 1.023 & 1.011 & 1.004 & 367 & 428 & 512 & -0.0743 \\
\hline 49 & 5234.625 & 1.044 & 1.025 & - & 332 & 520 & 697 & -0.2934 \\
\hline 49 & 5316.615 & 1.042 & 1.021 & 1.012 & 364 & 444 & 538 & -0.1777 \\
\hline 73 & 7515.831 & 1.029 & 1.029 & 1.022 & 236 & 473 & 644 & -0.3212 \\
\hline 73 & 7711.723 & 1.016 & 1.023 & 1.000 & 344 & 524 & 561 & -0.1118 \\
\hline 74 & 6149.258 & 1.028 & 1.029 & 1.013 & 360 & 544 & 633 & -0.2547 \\
\hline 74 & 6247.557 & 1.026 & 1.016 & 1.012 & 357 & 485 & 607 & -0.1796 \\
\hline 74 & 6416.919 & 1.027 & 1.015 & 1.000 & 392 & - & 560 & -0.1526 \\
\hline
\end{tabular}


M. L. Arias et al.: Fe II emission lines in Be stars, Online Material p 17

Table 5. continued.

\begin{tabular}{|c|c|c|c|c|c|c|c|c|}
\hline Multiplet & $\begin{array}{l}\lambda \\
\AA\end{array}$ & $I_{\mathrm{b}}$ & $I_{\mathrm{r}}$ & $I_{\mathrm{cd}}$ & $D_{\mathrm{p}}$ & $\begin{array}{l}D_{1 / 2} \\
\mathrm{~km} \mathrm{~s}^{-1} \\
\end{array}$ & $D_{1}$ & $\begin{array}{c}W_{\lambda} \\
\AA\end{array}$ \\
\hline \multicolumn{9}{|c|}{ HD 88661} \\
\hline 27 & 4233.172 & 1.037 & 1.060 & - & 341 & 459 & 544 & -0.3227 \\
\hline 27 & 4351.769 & 1.019 & 1.045 & - & 351 & 432 & 547 & -0.1734 \\
\hline 37 & 4629.339 & 1.033 & 1.012 & 0 & 373 & 0 & 577 & -0.1121 \\
\hline 38 & 4508.288 & 1.039 & 1.006 & 0 & 296 & 0 & 585 & -0.1385 \\
\hline 42 & 4923.927 & 1.018 & 1.054 & - & 392 & - & 479 & -0.1334 \\
\hline 42 & 5018.440 & 1.007 & 1.069 & - & 365 & - & 526 & -0.2065 \\
\hline 42 & 5169.033 & 1.028 & 1.075 & - & 336 & 421 & 575 & -0.3063 \\
\hline 48 & 5362.869 & 1.007 & 1.031 & - & 365 & - & 495 & -0.0729 \\
\hline 49 & 5197.577 & 1.007 & 1.034 & - & 417 & - & 497 & -0.1557 \\
\hline 49 & 5234.625 & 1.016 & 1.042 & - & 349 & - & 541 & -0.1669 \\
\hline 49 & 5276.002 & 1.021 & 1.039 & - & 393 & - & 506 & -0.1635 \\
\hline 49 & 5316.615 & 1.029 & 1.064 & - & 329 & 369 & 493 & -0.3013 \\
\hline 73 & 7515.831 & 1.035 & 1.034 & - & 286 & 403 & 509 & -0.3490 \\
\hline 74 & 6247.557 & 1.014 & 1.032 & - & 419 & 518 & 583 & -0.2423 \\
\hline \multicolumn{9}{|c|}{ HD 91465} \\
\hline 27 & 4233.172 & 1.087 & 1.033 & 1.017 & 252 & 390 & 563 & -0.2763 \\
\hline 27 & 4303.176 & 1.034 & 1.030 & 1.004 & 303 & 500 & 661 & -0.1631 \\
\hline 27 & 4351.769 & 1.051 & 1.029 & 1.000 & 288 & 404 & 600 & -0.1625 \\
\hline 27 & 4416.830 & 1.036 & 1.030 & 1.011 & 290 & 498 & 686 & -0.2019 \\
\hline 37 & 4555.893 & 1.063 & 1.020 & 1.001 & 237 & - & - & -0.1655 \\
\hline 37 & 4629.339 & 1.049 & 1.022 & 0.994 & 271 & 362 & 520 & -0.1138 \\
\hline 38 & 4549.474 & 1.064 & - & 1.000 & 104 & - & - & -0.1458 \\
\hline 38 & 4583.837 & 1.073 & 1.051 & 0.992 & 284 & 423 & 587 & -0.2545 \\
\hline 42 & 4923.927 & 1.094 & 1.050 & 1.000 & 287 & 406 & 592 & -0.3448 \\
\hline 42 & 5018.440 & 1.115 & 1.051 & 1.000 & 256 & 378 & 571 & -0.3643 \\
\hline 42 & 5169.033 & 1.108 & 1.056 & 1.006 & 246 & 375 & 576 & -0.3888 \\
\hline 48 & 5362.869 & 1.054 & 1.036 & 1.008 & 297 & 434 & 669 & -0.2423 \\
\hline 49 & 5197.577 & 1.054 & 1.033 & 1.002 & 279 & 391 & 575 & -0.2076 \\
\hline 49 & 5234.625 & 1.072 & 1.041 & 1.016 & 267 & 425 & 615 & -0.3342 \\
\hline 49 & 5276.002 & 1.071 & 1.025 & 1.000 & 249 & 316 & 474 & -0.1938 \\
\hline 49 & 5316.615 & 1.127 & 1.056 & 1.016 & 252 & 459 & 608 & -0.4766 \\
\hline 55 & 5534.847 & 1.042 & 1.014 & 1.000 & 288 & 359 & 497 & -0.1208 \\
\hline 73 & 7515.831 & 1.060 & 1.040 & 1.037 & 233 & 515 & 689 & -0.5881 \\
\hline 73 & 7711.723 & 1.056 & 1.028 & 1.001 & 273 & - & 476 & -0.2306 \\
\hline 74 & 6147.741 & - & - & - & - & - & - & -0.1954 \\
\hline 74 & 6149.258 & - & - & - & - & - & - & -0.1954 \\
\hline 74 & 6247.557 & 1.047 & 1.037 & 1.011 & 323 & 451 & 656 & -0.3189 \\
\hline 74 & 6416.919 & 1.024 & 1.011 & 1.000 & 349 & 480 & 586 & -0.1139 \\
\hline 74 & 6456.383 & 1.066 & 1.033 & 1.001 & 310 & 411 & 569 & -0.2792 \\
\hline \multicolumn{9}{|c|}{ HD 105435} \\
\hline 27 & 4233.172 & 1.048 & 1.034 & - & 208 & 352 & 407 & -0.1822 \\
\hline 27 & 4351.769 & 1.035 & 1.029 & - & 213 & 343 & 470 & -0.1381 \\
\hline 37 & 4555.893 & 1.026 & 1.024 & 1.014 & 153 & 323 & 381 & -0.1049 \\
\hline 37 & 4629.339 & 1.021 & 1.011 & 1.000 & 229 & 313 & 346 & -0.0428 \\
\hline 38 & 4508.288 & 1.017 & 1.009 & - & 278 & - & 354 & -0.0579 \\
\hline 38 & 4522.634 & 1.014 & 1.007 & 0 & 279 & 336 & 352 & -0.0250 \\
\hline 38 & 4549.474 & 1.042 & 1.028 & 1.017 & 261 & 372 & 429 & -0.1541 \\
\hline 38 & 4583.837 & 1.029 & 1.037 & 1.014 & 228 & 331 & 394 & -0.1166 \\
\hline 42 & 4923.927 & 1.026 & 1.048 & 1.010 & 207 & 319 & 380 & -0.1582 \\
\hline 42 & 5018.440 & 1.058 & 1.062 & 1.036 & 216 & 344 & 416 & -0.2992 \\
\hline 42 & 5169.033 & 1.052 & 1.047 & 1.028 & 212 & 330 & 398 & -0.2370 \\
\hline 48 & 5362.869 & 1.015 & 1.014 & 1.004 & 274 & 360 & 383 & -0.0617 \\
\hline 49 & 5197.577 & 1.027 & 1.027 & 1.017 & 242 & 342 & 383 & -0.1321 \\
\hline 49 & 5234.625 & 1.022 & 1.022 & 1.012 & 210 & 336 & 363 & -0.1139 \\
\hline 49 & 5276.002 & 1.037 & 1.022 & 1.018 & 232 & 325 & 406 & -0.1419 \\
\hline 49 & 5316.615 & 1.081 & 1.060 & 1.048 & 198 & 332 & 414 & -0.3554 \\
\hline
\end{tabular}


M. L. Arias et al.: Fe II emission lines in Be stars, Online Material p 18

Table 5. continued.

\begin{tabular}{|c|c|c|c|c|c|c|c|c|}
\hline Multiplet & $\begin{array}{l}\lambda \\
\AA \\
\AA\end{array}$ & $I_{\mathrm{b}}$ & $I_{\mathrm{r}}$ & $I_{\mathrm{cd}}$ & $D_{\mathrm{p}}$ & $\begin{array}{l}D_{1 / 2} \\
\mathrm{~km} \mathrm{~s}^{-1} \\
\end{array}$ & $D_{1}$ & $\begin{array}{c}W_{\lambda} \\
\AA\end{array}$ \\
\hline \multicolumn{9}{|c|}{ HD 110335} \\
\hline 27 & 4233.172 & 1.038 & 1.035 & 1.013 & 213 & 457 & 582 & -0.1938 \\
\hline 27 & 4303.176 & 1.018 & 1.020 & - & 294 & 423 & 490 & -0.0918 \\
\hline 27 & 4351.769 & 1.024 & 1.021 & 1.002 & 204 & 487 & 574 & -0.1175 \\
\hline 27 & 4416.830 & 1.016 & 1.017 & 1.005 & 231 & - & 744 & -0.1151 \\
\hline 37 & 4515.339 & 1.016 & 1.015 & 0.995 & 285 & 498 & 600 & -0.0809 \\
\hline 37 & 4555.893 & 1.035 & 1.024 & 1.010 & 210 & 308 & 367 & -0.1078 \\
\hline 37 & 4629.339 & 1.034 & 1.041 & 1.008 & 187 & 337 & 427 & -0.1438 \\
\hline 38 & 4508.288 & 1.012 & 1.019 & 0.998 & 252 & 506 & 604 & -0.0862 \\
\hline 38 & 4549.474 & 1.036 & 1.040 & 1.005 & 242 & 386 & 415 & -0.1387 \\
\hline 38 & 4583.837 & 1.040 & 1.043 & 0.999 & 281 & 466 & 529 & -0.2354 \\
\hline 42 & 4923.927 & 1.030 & 1.043 & 0.998 & 201 & 375 & 449 & -0.1908 \\
\hline 42 & 5018.440 & 1.046 & 1.048 & 1.000 & 202 & 398 & 532 & -0.2469 \\
\hline 42 & 5169.033 & 1.056 & 1.049 & 1.004 & 210 & 381 & 503 & -0.2678 \\
\hline 48 & 5362.869 & 1.017 & 1.020 & 1.002 & 255 & 478 & 584 & -0.1299 \\
\hline 49 & 5197.577 & 1.028 & 1.028 & 1.007 & 225 & 373 & 541 & -0.1510 \\
\hline 49 & 5234.625 & 1.029 & 1.026 & 1.006 & 207 & 410 & 526 & -0.1651 \\
\hline 49 & 5276.002 & 1.037 & 1.037 & 1.009 & 210 & 382 & 567 & -0.1995 \\
\hline 49 & 5316.615 & 1.048 & 1.052 & 0.997 & 203 & 336 & 505 & -0.2375 \\
\hline 74 & 6247.557 & 1.015 & 1.023 & 1.007 & 225 & 420 & 505 & -0.1276 \\
\hline 74 & 6416.919 & 1.014 & 1.011 & 1.001 & 301 & 510 & 563 & -0.0955 \\
\hline \multicolumn{9}{|c|}{ HD 112091} \\
\hline 27 & 4233.172 & 1.029 & 1.028 & 1.021 & 123 & 272 & 380 & -0.1001 \\
\hline 27 & 4351.769 & 1.016 & 1.017 & 1.004 & 136 & 251 & 287 & -0.0353 \\
\hline 38 & 4522.634 & 1.023 & 1.017 & 1.015 & 109 & 321 & 427 & -0.0928 \\
\hline 38 & 4549.474 & 1.026 & 1.029 & 1.014 & 109 & - & - & -0.1312 \\
\hline 38 & 4583.837 & 1.012 & 1.014 & 0.999 & 124 & 280 & 339 & -0.0345 \\
\hline 42 & 4923.927 & 1.020 & 1.023 & 1.012 & 142 & 260 & 335 & -0.0751 \\
\hline 42 & 5018.440 & 1.016 & 1.016 & 1.002 & 190 & 354 & 405 & -0.0757 \\
\hline 42 & 5169.033 & 1.031 & 1.028 & 1.009 & 173 & 342 & 432 & -0.1402 \\
\hline 49 & 5197.577 & 1.014 & 1.017 & 1.013 & 133 & 271 & 390 & -0.0720 \\
\hline 49 & 5234.625 & 1.017 & 1.013 & - & 111 & 346 & 399 & -0.0763 \\
\hline 49 & 5276.002 & 1.020 & 1.012 & 1.004 & 149 & 290 & 345 & -0.0666 \\
\hline 49 & 5316.615 & 1.049 & 1.047 & 1.026 & 149 & 302 & 432 & -0.2412 \\
\hline \multicolumn{9}{|c|}{ HD 120991} \\
\hline 27 & 4233.172 & 1.066 & 1.085 & 1.060 & 73 & 129 & 236 & -0.1592 \\
\hline 27 & 4303.176 & 1.046 & 1.019 & 1.018 & 83 & 127 & 190 & -0.0576 \\
\hline 27 & 4351.769 & 1.041 & 1.084 & 1.036 & 93 & 153 & 182 & -0.1178 \\
\hline 28 & 4178.862 & 1.083 & - & - & 76 & - & 236 & -0.0927 \\
\hline 37 & 4515.339 & 1.047 & 1.045 & 1.037 & 74 & 131 & 185 & -0.0879 \\
\hline 37 & 4520.224 & 1.036 & 1.035 & 1.031 & 77 & 127 & 165 & -0.0642 \\
\hline 37 & 4534.168 & 1.021 & 1.024 & 1.009 & 89 & 121 & 167 & -0.0328 \\
\hline 37 & 4555.893 & 1.061 & 1.050 & 1.040 & 67 & 124 & 163 & -0.0933 \\
\hline 38 & 4508.288 & 1.041 & 1.067 & 1.037 & 77 & 132 & 155 & -0.1031 \\
\hline 38 & 4522.634 & 1.048 & 1.041 & 1.035 & 62 & 124 & 157 & -0.0810 \\
\hline 38 & 4549.474 & 1.066 & 1.076 & 1.051 & 74 & 134 & 185 & -0.1373 \\
\hline 38 & 4583.837 & 1.065 & 1.054 & 1.047 & 67 & 119 & 143 & -0.1029 \\
\hline 41 & 5284.109 & - & - & - & - & - & - & -0.0416 \\
\hline 42 & 4923.927 & 1.085 & 1.068 & 1.056 & 86 & 127 & 154 & -0.1413 \\
\hline 42 & 5018.440 & 1.067 & 1.088 & 1.063 & 55 & 118 & 152 & -0.1446 \\
\hline 42 & 5169.033 & 1.091 & 1.076 & 1.061 & 83 & 126 & 163 & -0.1679 \\
\hline 43 & 4731.453 & 1.022 & 1.024 & 1.010 & 99 & 124 & 154 & -0.0370 \\
\hline 48 & 5362.869 & 1.049 & 1.047 & 1.036 & 83 & 121 & 181 & -0.0995 \\
\hline 49 & 5197.577 & 1.049 & 1.059 & 1.040 & 78 & 125 & 174 & -0.1087 \\
\hline 49 & 5234.625 & 1.082 & 1.047 & 1.044 & 73 & 115 & 155 & -0.1226 \\
\hline 49 & 5276.002 & 1.067 & 1.048 & 1.047 & 66 & 122 & 146 & -0.1165 \\
\hline 49 & 5316.615 & 1.125 & 1.132 & 1.112 & 56 & 124 & 182 & -0.2680 \\
\hline 49 & 5325.553 & 1.022 & 1.021 & 1.013 & 101 & 126 & 148 & -0.0359 \\
\hline
\end{tabular}


M. L. Arias et al.: Fe II emission lines in Be stars, Online Material p 19

Table 5. continued.

\begin{tabular}{|c|c|c|c|c|c|c|c|c|}
\hline Multiplet & $\begin{array}{l}\lambda \\
\AA\end{array}$ & $I_{\mathrm{b}}$ & $I_{\mathrm{r}}$ & $I_{\mathrm{cd}}$ & $D_{\mathrm{p}}$ & $\frac{D_{1 / 2}}{\mathrm{~km} \mathrm{~s}^{-1}}$ & $D_{1}$ & $\begin{array}{c}W_{\lambda} \\
\AA\end{array}$ \\
\hline 55 & 5534.847 & 1.052 & 1.031 & 1.022 & 82 & 122 & 156 & -0.0815 \\
\hline 73 & 7462.407 & 0 & 0 & 0 & 0 & 0 & 256 & -0.1158 \\
\hline 73 & 7515.831 & 0 & 0 & 0 & 0 & 0 & 262 & -0.2902 \\
\hline 73 & 7711.723 & 1.067 & 1.056 & 1.048 & 67 & 124 & 159 & -0.1826 \\
\hline 74 & 6147.741 & - & - & - & - & - & - & -0.0582 \\
\hline 74 & 6149.258 & - & - & - & - & - & - & -0.0582 \\
\hline 74 & 6238.392 & 1.023 & 1.035 & 1.025 & 67 & 119 & 151 & -0.0757 \\
\hline 74 & 6247.557 & 1.056 & 1.080 & 1.048 & 79 & 170 & 263 & -0.2217 \\
\hline 74 & 6416.919 & 1.022 & 1.025 & 1.015 & 77 & 118 & 156 & -0.0492 \\
\hline 74 & 6456.383 & 1.092 & 1.075 & 1.063 & 66 & 123 & 196 & -0.2143 \\
\hline \multicolumn{9}{|c|}{ HD 124367} \\
\hline 27 & 4233.172 & 1.045 & 1.042 & 1.021 & 171 & 427 & 672 & -0.2342 \\
\hline 27 & 4273.326 & 1.045 & - & - & 74 & - & - & -0.1209 \\
\hline 27 & 4351.769 & 1.033 & 1.059 & 1.018 & 271 & 466 & 533 & -0.2748 \\
\hline 27 & 4416.830 & 1.026 & 1.020 & 1.011 & 318 & 596 & 714 & -0.1599 \\
\hline 37 & 4555.893 & 1.020 & 1.018 & 1.000 & 224 & 338 & 485 & -0.0737 \\
\hline 37 & 4629.339 & 1.020 & 1.025 & 1.001 & 222 & 410 & 575 & -0.1089 \\
\hline 38 & 4549.474 & 1.032 & 1.036 & 1.012 & 210 & 435 & 630 & -0.1800 \\
\hline 38 & 4583.837 & 1.032 & 1.032 & 1.009 & 272 & 458 & 586 & -0.1956 \\
\hline 42 & 4923.927 & 1.028 & 1.032 & 1.002 & 239 & 455 & 568 & -0.1613 \\
\hline 42 & 5018.440 & 1.046 & 1.072 & 1.008 & 237 & 529 & 633 & -0.3856 \\
\hline 42 & 5169.033 & 1.051 & 1.071 & 1.018 & 192 & 416 & 714 & -0.4015 \\
\hline 48 & 5362.869 & 1.025 & 1.021 & 1.005 & 267 & 507 & 618 & -0.1544 \\
\hline 49 & 5197.577 & 1.019 & 1.025 & 1.002 & 248 & 439 & 560 & -0.1332 \\
\hline 49 & 5234.625 & 1.056 & 1.053 & 1.030 & 209 & 461 & 729 & -0.4179 \\
\hline 49 & 5276.002 & 1.023 & 1.028 & 0.999 & 213 & 354 & 626 & -0.1422 \\
\hline 49 & 5316.615 & 1.094 & 1.094 & 1.046 & 186 & 420 & 735 & -0.6153 \\
\hline 55 & 5534.847 & 1.040 & 1.031 & 1.022 & 204 & 612 & 715 & -0.2919 \\
\hline 73 & 7462.407 & - & - & - & - & - & - & -0.2088 \\
\hline 73 & 7515.831 & 1.026 & 1.026 & 1.008 & 246 & 381 & 558 & -0.1915 \\
\hline 73 & 7711.723 & 1.034 & 1.041 & 1.015 & 188 & 321 & 405 & -0.2688 \\
\hline 74 & 6147.741 & - & - & - & - & - & - & -0.1096 \\
\hline 74 & 6149.258 & - & - & - & - & - & - & -0.1096 \\
\hline 74 & 6247.557 & 1.028 & 1.032 & 1.016 & 243 & 479 & 653 & -0.2384 \\
\hline 74 & 6456.383 & 1.037 & 1.041 & 1.016 & 261 & 438 & 540 & -0.3006 \\
\hline \multicolumn{9}{|c|}{ HD 148184} \\
\hline 27 & 4173.461 & 1.080 & 1.077 & 1.066 & 91 & 226 & 364 & -0.265 \\
\hline 27 & 4233.172 & 1.120 & 1.146 & 1.114 & 67 & 191 & 415 & -0.373 \\
\hline 27 & 4351.769 & 1.045 & 1.068 & 1.048 & 88 & 142 & 316 & -0.278 \\
\hline 37 & 4515.339 & 1.062 & 1.068 & 0 & 94 & 188 & 315 & -0.192 \\
\hline 37 & 4534.168 & 1.041 & 1.038 & 1.032 & 119 & 244 & 351 & -0.140 \\
\hline 37 & 4555.893 & 1.132 & 1.134 & 1.122 & 82 & 210 & 380 & -0.390 \\
\hline 37 & 4629.339 & 1.084 & 1.075 & 1.063 & 82 & 173 & 262 & -0.231 \\
\hline 38 & 4508.288 & 1.067 & 1.082 & 1.060 & 83 & 218 & 389 & -0.259 \\
\hline 38 & 4549.474 & 1.104 & 1.143 & 1.097 & 94 & 189 & 316 & -0.353 \\
\hline 38 & 4583.837 & 1.129 & 1.116 & 1.101 & 84 & 186 & 373 & -0.379 \\
\hline 41 & 5284.109 & 0 & 1.049 & 0 & 0 & 187 & 273 & -0.101 \\
\hline 42 & 4924.927 & 1.092 & 1.135 & 1.081 & 86 & 168 & 333 & -0.330 \\
\hline 42 & 5018.440 & 1.163 & 1.151 & 1.133 & 84 & 163 & 275 & -0.444 \\
\hline 42 & 5169.033 & 1.160 & 1.157 & 1.129 & 87 & 180 & 310 & -0.508 \\
\hline 43 & 4731.453 & 1.036 & 1.044 & 0 & 120 & 219 & 339 & -0.141 \\
\hline 48 & 5362.869 & 1.065 & 1.079 & 1.060 & 94 & 194 & 315 & -0.245 \\
\hline 49 & 5197.577 & 1.086 & 1.095 & 1.072 & 84 & 182 & 343 & -0.290 \\
\hline 49 & 5234.625 & 1.082 & 1.102 & 1.073 & 89 & 180 & 296 & -0.281 \\
\hline 49 & 5276.002 & 1.099 & 1.109 & 1.090 & 82 & 181 & 248 & -0.322 \\
\hline 49 & 5316.615 & 1.209 & 1.221 & 1.177 & 90 & 178 & 320 & -0.326 \\
\hline 55 & 5534.847 & 1.056 & 1.068 & 1.045 & 94 & 123 & 278 & -0.287 \\
\hline
\end{tabular}


M. L. Arias et al.: Fe II emission lines in Be stars, Online Material p 20

Table 5. continued.

\begin{tabular}{|c|c|c|c|c|c|c|c|c|}
\hline Multiplet & $\begin{array}{l}\lambda \\
\AA\end{array}$ & $I_{\mathrm{b}}$ & $I_{\mathrm{r}}$ & $I_{\mathrm{cd}}$ & $D_{\mathrm{p}}$ & $\frac{D_{1 / 2}}{\mathrm{~km} \mathrm{~s}^{-1}}$ & $D_{1}$ & $\begin{array}{c}W_{\lambda} \\
\AA\end{array}$ \\
\hline 73 & 7462.407 & 1.032 & 1.035 & 1.023 & 112 & 202 & 286 & -0.149 \\
\hline 73 & 7515.831 & 1.098 & 0 & 0 & 0 & 183 & 405 & -0.470 \\
\hline 73 & 7711.723 & 1.086 & 1.099 & 1.074 & 67 & 162 & 298 & -0.389 \\
\hline 74 & 6416.919 & 1.024 & 1.029 & 1.014 & 112 & 210 & 289 & -0.098 \\
\hline 74 & 6456.383 & 1.108 & 1.131 & 1.099 & 100 & 207 & 327 & -0.522 \\
\hline \multicolumn{9}{|c|}{ HD 157042} \\
\hline 27 & 4233.172 & 1.033 & 1.018 & 1.016 & 494 & 656 & 782 & -0.1934 \\
\hline 27 & 4351.769 & 1.019 & 1.015 & 1.003 & 467 & 586 & 661 & -0.1024 \\
\hline 38 & 4583.837 & 1.031 & 1.016 & 0 & 486 & 536 & 637 & -0.2093 \\
\hline 42 & 4923.927 & 1.028 & 1.025 & - & 482 & 594 & 714 & -0.1393 \\
\hline 42 & 5018.440 & 1.033 & 1.036 & - & 406 & 614 & 704 & -0.2745 \\
\hline 42 & 5169.033 & 1.041 & 1.024 & - & 431 & 599 & 689 & -0.2311 \\
\hline 49 & 5316.615 & 1.043 & 1.013 & - & 484 & 572 & 668 & -0.2160 \\
\hline \multicolumn{9}{|c|}{ HD 158427} \\
\hline 27 & 4233.172 & 1.041 & 1.021 & 1.011 & 244 & 431 & 628 & -0.1642 \\
\hline 27 & 4351.769 & 1.015 & 1.025 & 1.001 & 250 & 452 & 583 & -0.0947 \\
\hline 27 & 4416.830 & 1.012 & 1.017 & 1.004 & 237 & 440 & 567 & -0.0723 \\
\hline 37 & 4629.339 & 1.035 & 1.025 & 1.006 & 289 & 434 & 528 & -0.1415 \\
\hline 38 & 4583.837 & 1.052 & 1.061 & 1.034 & 223 & 424 & 646 & -0.3458 \\
\hline 42 & 4923.927 & 1.038 & 1.043 & 1.000 & 266 & 491 & 685 & -0.2305 \\
\hline 42 & 5018.440 & 1.055 & 1.070 & 1.014 & 257 & 448 & 668 & -0.3694 \\
\hline 42 & 5169.033 & 1.061 & 1.056 & 1.015 & 253 & 492 & 651 & -0.3831 \\
\hline 48 & 5362.869 & 1.020 & 1.024 & 1.011 & 281 & 549 & 613 & -0.1521 \\
\hline 49 & 5197.577 & 1.036 & 1.030 & 1.014 & 263 & 454 & 726 & -0.2274 \\
\hline 49 & 5234.625 & 1.029 & 1.029 & 1.011 & 223 & 449 & 580 & -0.1736 \\
\hline 49 & 5276.002 & 1.030 & 1.021 & 1.005 & 254 & 435 & 604 & -0.1527 \\
\hline 49 & 5316.615 & 1.081 & 1.067 & 1.040 & 233 & 442 & 638 & -0.5056 \\
\hline 73 & 7515.831 & 1.063 & 1.064 & 0 & 227 & 500 & 738 & -0.7633 \\
\hline 73 & 7711.723 & 1.026 & 1.038 & 1.014 & 244 & 432 & 537 & -0.3073 \\
\hline 74 & 6247.557 & 1.012 & 1.014 & 1.005 & 213 & 432 & 554 & -0.0915 \\
\hline
\end{tabular}

$I_{\mathrm{b}}, I_{\mathrm{r}}$, and $I_{\mathrm{cd}}=$ intensities of the blue, red peak, and central depression in the two-peaked emission profiles in units of the local continuum; $D_{\mathrm{p}}$, $D_{1 / 2}$, and $D_{1}=$ separation of emission peaks, width of lines at half intensity and at intensity $I / I_{\mathrm{c}}=1.0$ in velocity units; $W_{\lambda}$ : equivalent width. 
M. L. Arias et al.: Fe II emission lines in Be stars, Online Material p 21

Table 6. Measurements of the observed hydrogen Balmer emission lines.

\begin{tabular}{|c|c|c|c|c|c|c|c|c|c|}
\hline HD & $I_{\mathrm{p}}$ & $I_{\mathrm{b}}$ & $I_{\mathrm{r}}$ & $D_{\mathrm{p}}$ & $\frac{D_{1 / 2}}{\mathrm{~m} \mathrm{~s}^{-1}}$ & $D_{1}$ & $\begin{array}{c}W_{\lambda} \\
\AA \\
\end{array}$ & $\begin{array}{c}W_{\mathrm{cd}} \\
\AA\end{array}$ & Npeaks \\
\hline \multicolumn{10}{|c|}{$\mathrm{H} \alpha$} \\
\hline 41335 & 0.000 & 4.188 & 3.593 & 197 & 464 & 1364 & -31.155 & 0.331 & 2 \\
\hline 45725 & 0.000 & 4.136 & 4.236 & 147 & 428 & 1446 & -33.197 & 0.361 & 2 \\
\hline 48917 & 0.000 & 3.725 & 3.795 & 107 & 337 & 1433 & -23.665 & 0.110 & 2 \\
\hline 50013 & 5.691 & 0.000 & 0.000 & 0 & 356 & 823 & -28.292 & 0.000 & 1 \\
\hline 56139 & 3.465 & 0.000 & 0.000 & 0 & 205 & 625 & -10.731 & 0.000 & 1 \\
\hline 58978 & 1.882 & 1.714 & 1.849 & 354 & 608 & 1500 & -12.105 & 0.000 & 3 \\
\hline 63462 & 0.000 & 2.177 & 1.992 & 182 & 499 & 1171 & -12.623 & 0.117 & 2 \\
\hline 88661 & 0.000 & 4.778 & 3.999 & 111 & 288 & 924 & -23.970 & 0.156 & 2 \\
\hline 91465 & 0.000 & 4.073 & 4.119 & 95 & 379 & 1385 & -28.726 & 0.041 & 2 \\
\hline 105435 & - & - & - & - & - & - & - & - & - \\
\hline 110335 & 0.000 & 3.690 & 3.639 & 95 & 299 & 809 & -18.628 & 0.146 & 2 \\
\hline 112091 & 0.000 & 5.482 & 5.510 & 62 & 252 & 755 & -26.243 & 0.031 & 2 \\
\hline 120991 & 4.587 & 0.000 & 0.000 & 0 & 170 & 872 & -16.174 & 0.000 & 1 \\
\hline 124367 & 0.000 & 5.960 & 5.980 & 92 & 319 & 1324 & -39.113 & 0.070 & 2 \\
\hline 148184 & 11.000 & 0.000 & 0.000 & 0 & 151 & 1214 & -42.838 & 0.000 & 1 \\
\hline 157042 & - & - & - & - & - & - & - & - & - \\
\hline 158427 & 0.000 & 4.694 & 4.477 & 124 & 389 & 1522 & -33.478 & 0.121 & 2 \\
\hline 164284 & 0.000 & 7.091 & 7.036 & 86 & 304 & 1031 & -44.220 & 0.070 & 2 \\
\hline \multicolumn{10}{|c|}{$\mathrm{H} \beta$} \\
\hline 41335 & 0.000 & 1.540 & 1.502 & 221 & 376 & 755 & -2.901 & 0.294 & 2 \\
\hline 45725 & 0.000 & 1.589 & 1.596 & 219 & 380 & 648 & -3.155 & 0.457 & 2 \\
\hline 48917 & 0.000 & 1.423 & 1.407 & 188 & 316 & 653 & -2.091 & 0.165 & 2 \\
\hline 50013 & 0.000 & 1.629 & 1.625 & 162 & 307 & 866 & -3.325 & 0.101 & 2 \\
\hline 56139 & 0.000 & 1.359 & 1.363 & 45 & 168 & 320 & -1.008 & 0.004 & 2 \\
\hline 58978 & 0.000 & 1.158 & 1.157 & 331 & 478 & 774 & -0.956 & 0.299 & 2 \\
\hline 63462 & 0.000 & 1.270 & 1.220 & 249 & 456 & 783 & -1.778 & 0.094 & 2 \\
\hline 88661 & 0.000 & 1.719 & 1.554 & 144 & 280 & 836 & -3.084 & 0.170 & 2 \\
\hline 91465 & 0.000 & 1.568 & 1.461 & 158 & 344 & 1094 & -3.046 & 0.140 & 2 \\
\hline 105435 & 0.000 & 1.934 & 1.715 & 117 & 251 & 730 & -3.486 & 0.117 & 2 \\
\hline 110335 & 0.000 & 1.398 & 1.392 & 176 & 314 & 625 & -1.770 & 0.247 & 2 \\
\hline 112091 & 0.000 & 1.418 & 1.417 & 135 & 290 & 573 & -1.984 & 0.087 & 2 \\
\hline 120991 & 2.147 & 0.000 & 0.000 & 0 & 108 & 344 & -2.204 & 0.000 & 1 \\
\hline 124367 & 0.000 & 1.547 & 1.571 & 208 & 349 & 890 & -3.404 & 0.192 & 2 \\
\hline 148184 & 2.981 & 0.000 & 0.000 & 0 & 133 & 646 & -4.912 & 0.000 & 1 \\
\hline 157042 & 0.000 & 1.283 & 1.443 & 220 & 391 & 802 & -2.203 & 0.256 & 2 \\
\hline 158427 & 0.000 & 1.492 & 1.429 & 192 & 378 & 825 & -2.798 & 0.177 & 2 \\
\hline 164284 & 0.000 & 1.681 & 1.769 & 153 & 320 & 740 & -3.704 & 0.184 & 2 \\
\hline \multicolumn{10}{|c|}{$\mathrm{H} \gamma$} \\
\hline 41335 & 0.000 & 1.138 & 1.152 & 222 & 379 & 688 & -0.623 & 0.181 & 2 \\
\hline 45725 & 0.000 & 1.160 & 1.166 & 232 & 366 & 532 & -0.498 & 0.318 & 2 \\
\hline 48917 & 0.000 & 1.122 & 1.092 & 217 & 303 & 687 & -0.366 & 0.163 & $2-3$ \\
\hline 50013 & 0.000 & 1.177 & 1.182 & 166 & 308 & 430 & -0.704 & 0.099 & 2 \\
\hline 56139 & 0.000 & 1.114 & 1.112 & 55 & 167 & 289 & -0.280 & 0.005 & 2 \\
\hline 58978 & 0.000 & 1.094 & 1.085 & 312 & 471 & 611 & -0.457 & 0.202 & 2 \\
\hline 63462 & 0.000 & 1.112 & 1.082 & 286 & 501 & 779 & -0.597 & 0.089 & 2 \\
\hline 88661 & 0.000 & 1.195 & 1.168 & 163 & 284 & 633 & -0.687 & 0.127 & 2 \\
\hline 91465 & 0.000 & 1.152 & 1.107 & 180 & 362 & 663 & -0.585 & 0.082 & 2 \\
\hline 105435 & 0.000 & 1.243 & 1.187 & 139 & 260 & 469 & -0.753 & 0.080 & 2 \\
\hline 110335 & 0.000 & 1.142 & 1.131 & 194 & 323 & 522 & -0.497 & 0.143 & 2 \\
\hline 112091 & 0.000 & 1.069 & 1.061 & 187 & 284 & 413 & -0.201 & 0.073 & 2 \\
\hline 120991 & 1.520 & 0.000 & 0.000 & 0 & 110 & 273 & -0.872 & 0.000 & 1 \\
\hline 124367 & 0.000 & 1.095 & 1.097 & 204 & 331 & 560 & -0.384 & 0.115 & 2 \\
\hline 148184 & 0.000 & 1.694 & 1.639 & 33 & 154 & 445 & -1.619 & 0.000 & 2 \\
\hline 157042 & 0.000 & 1.085 & 1.114 & 269 & 390 & 555 & -0.365 & 0.186 & 2 \\
\hline 158427 & 0.000 & 1.086 & 1.069 & 223 & 360 & 507 & -0.307 & 0.121 & 2 \\
\hline 164367 & 0.000 & 1.172 & 1.216 & 262 & 311 & 520 & -0.772 & 0.120 & 2 \\
\hline
\end{tabular}


M. L. Arias et al.: Fe II emission lines in Be stars, Online Material p 22

Table 6. continued.

\begin{tabular}{|c|c|c|c|c|c|c|c|c|c|}
\hline HD & $I_{\mathrm{p}}$ & $I_{\mathrm{b}}$ & $I_{\mathrm{r}}$ & $D_{\mathrm{p}}$ & $\frac{D_{1 / 2}}{\mathrm{~m} \mathrm{~s}^{-1}}$ & $D_{1}$ & $\begin{array}{c}W_{\lambda} \\
\AA\end{array}$ & $\begin{array}{c}W_{\mathrm{cd}} \\
\AA\end{array}$ & Npeaks \\
\hline \multicolumn{10}{|c|}{$\overline{\mathrm{H} \delta}$} \\
\hline 41335 & 0.000 & 1.063 & 1.074 & 251 & 0 & 621 & -0.286 & 0.124 & 2 \\
\hline 45725 & 0.000 & 1.041 & 1.031 & 289 & 0 & 0 & -0.089 & 0.307 & 2 \\
\hline 48917 & 0.000 & 1.109 & 1.055 & 244 & 404 & 688 & -0.364 & 0.150 & $2-3$ \\
\hline 50013 & 0.000 & 1.098 & 1.059 & 197 & 317 & 477 & -0.259 & 0.084 & 2 \\
\hline 56139 & 0.000 & 1.048 & 1.048 & 84 & 176 & 287 & -0.113 & 0.009 & 2 \\
\hline 58978 & 0.000 & 1.043 & 1.050 & 307 & 490 & 560 & -0.232 & 0.082 & 2 \\
\hline 63462 & 0.000 & 1.086 & 1.039 & 358 & 521 & 732 & -0.320 & 0.123 & 2 \\
\hline 88661 & 0.000 & 1.067 & 1.080 & 195 & 313 & 599 & -0.265 & 0.088 & 2 \\
\hline 91465 & 0.000 & 1.052 & 1.011 & 200 & 0 & 381 & -0.085 & 0.046 & 2 \\
\hline 105435 & 0.000 & 1.090 & 1.070 & 155 & 276 & 458 & -0.255 & 0.064 & 2 \\
\hline 110335 & 0.000 & 1.076 & 1.070 & 209 & 377 & 632 & -0.299 & 0.099 & 2 \\
\hline 110335 & 0.000 & 1.076 & 1.070 & 209 & 377 & 632 & -0.299 & 0.099 & 2 \\
\hline 112091 & 0.000 & 1.050 & 1.039 & 204 & 356 & 461 & -0.155 & 0.054 & 2 \\
\hline 120991 & 1.310 & 0.000 & 0.000 & 0 & 121 & 263 & -0.539 & 0.000 & 1 \\
\hline 124367 & 0.000 & 1.037 & 1.030 & 208 & 317 & 433 & -0.096 & 0.051 & 2 \\
\hline 148184 & 0.000 & 1.379 & 1.339 & 44 & 171 & 481 & -0.953 & 0.000 & 2 \\
\hline 157042 & 0.000 & 1.070 & 1.062 & 273 & 399 & 555 & -0.261 & 0.097 & 2 \\
\hline 158427 & 0.000 & 1.050 & 1.040 & 238 & 363 & 563 & -0.165 & 0.064 & 2 \\
\hline 164284 & 0.000 & 1.054 & 1.061 & 207 & 324 & 505 & -0.207 & 0.079 & 2 \\
\hline
\end{tabular}

$I_{\mathrm{p}}, I_{\mathrm{b}}$, and $I_{\mathrm{r}}=$ intensities of the peak of one-peaked line profiles, the blue and red peak intensities of two-peaked line profiles; $D_{\mathrm{p}}, D_{1 / 2}$, and $D_{1}=$ separation of emission peaks, width of lines at half intensity and at $I / I_{\mathrm{c}}=1.0$ in velocity units; $W_{\lambda}=$ equivalent width; $W_{\mathrm{cd}}=$ equivalent width of the central depression; Npeaks $=$ number of emission peaks. 
M. L. Arias et al.: Fe II emission lines in Be stars, Online Material p 23

Table 7. Atomic data of the sudied Fe II lines.

\begin{tabular}{ccccc}
\hline \hline Multiplet & $\lambda_{\mathrm{o}}$ & log gf & $\mathrm{E}_{l}$ & $\mathrm{E}_{u}$ \\
\hline 27 & 4128.748 & -3.470 & 2.583 & 5.585 \\
27 & 4173.461 & -2.513 & 2.583 & 5.553 \\
27 & 4233.172 & -1.836 & 2.583 & 5.511 \\
27 & 4273.326 & -3.258 & 2.704 & 5.605 \\
27 & 4303.176 & -2.443 & 2.704 & 5.585 \\
27 & 4351.769 & -2.130 & 2.704 & 5.553 \\
27 & 4385.387 & -2.542 & 2.778 & 5.605 \\
27 & 4416.830 & -2.534 & 2.778 & 5.585 \\
28 & 4178.862 & -2.785 & 2.583 & 5.549 \\
28 & 4258.154 & -3.799 & 2.704 & 5.615 \\
28 & 4296.572 & -3.198 & 2.704 & 5.589 \\
37 & 4489.183 & -3.422 & 2.828 & 5.589 \\
37 & 4491.405 & -2.684 & 2.856 & 5.615 \\
37 & 4515.339 & -2.467 & 2.844 & 5.589 \\
37 & 4520.224 & -2.983 & 2.807 & 5.549 \\
37 & 4534.168 & -3.253 & 2.856 & 5.589 \\
37 & 4555.893 & -2.325 & 2.828 & 5.549 \\
37 & 4582.835 & -3.094 & 2.844 & 5.549 \\
37 & 4629.339 & -2.306 & 2.807 & 5.484 \\
37 & 4666.758 & -3.221 & 2.828 & 5.484 \\
38 & 4508.288 & -2.312 & 2.856 & 5.605 \\
38 & 4522.634 & -2.119 & 2.844 & 5.585 \\
38 & 4541.524 & -2.847 & 2.856 & 5.585 \\
38 & 4549.474 & -1.957 & 2.828 & 5.553 \\
38 & 4576.340 & -2.822 & 2.844 & 5.553 \\
38 & 4583.837 & -1.802 & 2.807 & 5.511 \\
43 & 4731.453 & -3.053 & 2.891 & 5.511 \\
42 & 4923.927 & -1.559 & 2.891 & 5.408 \\
42 & 5018.440 & -1.400 & 2.891 & 5.361 \\
42 & 5169.033 & -1.303 & 2.891 & 5.289 \\
49 & 5197.577 & -2.233 & 3.230 & 5.615 \\
49 & 5234.625 & -2.151 & 3.221 & 5.589 \\
49 & 5254.929 & -3.227 & 3.230 & 5.589 \\
49 & 5276.002 & -2.073 & 3.199 & 5.549 \\
49 & 5316.615 & -1.930 & 3.153 & 5.484 \\
49 & 5325.553 & -3.220 & 3.221 & 5.549 \\
49 & 5425.257 & -3.372 & 3.199 & 5.484 \\
41 & 5284.109 & -3.299 & 2.891 & 5.237 \\
48 & 5264.812 & -3.303 & 3.230 & 5.585 \\
48 & 5316.784 & -2.908 & 3.221 & 5.553 \\
48 & 5362.869 & -2.739 & 3.199 & 5.511 \\
55 & 5534.847 & -2.996 & 3.245 & 5.484 \\
74 & 6147.741 & -2.721 & 3.889 & 5.905 \\
74 & 6149.258 & -2.724 & 3.889 & 5.905 \\
74 & 6238.392 & -2.630 & 3.889 & 5.876 \\
74 & 6247.557 & -2.329 & 3.892 & 5.876 \\
74 & 6416.919 & -2.740 & 3.892 & 5.823 \\
74 & 6456.383 & -2.075 & 3.903 & 5.823 \\
40 & 6532.628 & -3.708 & 2.891 & 4.818 \\
43 & 7462.407 & -2.734 & 3.892 & 5.553 \\
73 & 7515.831 & -3.432 & 3.903 & 5.553 \\
73 & 7711.723 & -2.543 & 3.903 & 5.511 \\
\hline & & & & \\
\hline
\end{tabular}

